\title{
AN INTEGRATED BIOINFORMATICS APPROACH TO THE DISCOVERY OF $C I S$-REGULATORY ELEMENTS INVOLVED IN PLANT GRAVITROPIC SIGNAL TRANSDUCTION
}

\author{
X. Liang, ${ }^{*}$ K. Shen, ${ }^{* *, * * *}$ J. Lichtenberg, ${ }^{*}$ S.E. Wyatt, ${ }^{* *},{ }^{* * *}$ and L.R. Welch*,***,****
}

\begin{abstract}
Gravity is a common stimulus affecting plant growth and development, from seed germination to positioning of flowers for pollination and seeds for dispersal. Classic models of plant gravitropism have revolved around biophysical perception of the gravity stimulus and the effects of plant growth regulators on the growth response. Transcriptional regulation of the gravitropic mechanism has been largely ignored. The aim of this experiment is to identify putative regulatory functional elements, including transcription factor binding sites and cis-regulatory modules involved in gravitropic signal transduction.

In this article, we detailed a strategy to identify putative cis-regulatory elements by analyzing gene expression data from microarray experiments. Genes involved in the gravitropic perceptionresponse pathway were identified based on their changes in expression level after gravity stimulation. Genes were clustered according to their expression patterns (transcriptional regulation profiles), and gene promoter were analyzed using genomics regulatory analysis software to identify candidate cis-regulatory elements and cis-regulatory modules.

Analysis of the microarray data indicated that 154 genes were involved in the gravitropic response. The genes were grouped into 9 clusters based on expression profile similarities. An analysis of the promoters of the 154 genes resulted in the identification of 32 putative regulatory elements and 55 putative regulatory modules. Some of the elements are associated with individual clusters and other elements are associated with multiple clusters, potentially indicating elements involved in specific and in general gravitropic response processes, respectively.
\end{abstract}

\section{Key Words}

Gravitropism, motif discovery, regulatory genomics, module discovery, gene expression

* School of Electrical Engineering and Computer Science, Ohio University, Athens, Ohio, USA

** Department of Environmental and Plant Biology, Ohio University, Athens, Ohio, USA

*** Molecular and Cellular Biology Program, Ohio University, Athens, Ohio, USA

**** Biomedical Engineering Program, Ohio University, Athens, Ohio, USA; e-mail:

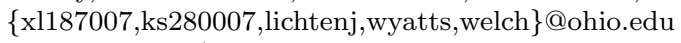

(paper no. 210-1013)

\section{Introduction}

Gravitropism refers to the plant growth response in the Earth's gravity field. Plants use gravity to control everything from the direction of growth of an emerging seedling, to the positioning of plant organs (stems, branches, primary and lateral roots, flowers and seed pods). Simplistically, gravitropism has been broken down into three steps: perception, signal transduction and growth response [1]. Commonly, the gravitational stimulus is believed to be sensed by dense organelles: statoliths. Statoliths, often amyloplasts full of starch, are located in specific cell layers (the columella cells of the root cap and in the starch sheath surrounding the vascular tissue in the shoots) that are known to control the gravitropic response (reviewed in [2]). When a plant is re-positioned relative to the gravity field, the statoliths "settle" on the new physical "bottom" of the cell, initiating a signal transduction cascade. Although in existence for over 100 years, the Starch-Statolith hypothesis does not tell the full story. Mutants lacking starch-filled statoliths also respond to gravity, indicating that additional mechanisms must be involved $[3,4]$. The signal transduction phase of the gravitropic pathway has been dominated by the movement and redistribution of the plant growth regulator auxin. However, how statolith sedimentation directs redistribution of auxin is still largely unknown. More recently, research has identified other potential aspects of the signal transduction phase (for review see [5]). Potential roles for cytoplasmic $\mathrm{pH}[6]$, cytoskeletal rearrangements (for review see [7]), inositol 1, 4, 5triphosphate $[8,9]$, and reactive oxygen species $[10,11]$ have all been proposed, but how these fit into the pathway is not clear. A plant's response to the gravitropic stimulus is a growth response that results in organ curvature to maintain the organ's original position to the gravity field. This curvature is the result of the different elongation of the cells within the elongation zone. The response leads to cell expansion, cell wall synthesis, and other physiologic 
events (for review see [12]).

Most gravitropic experiments have focused on the biophysical movement of statoliths, imaging of proton and calcium gradients, or the physiological interactions and responses involved with auxin and the growth response. Physiological experiments and mutant analyses have provided the majority of the data. Little has been done on a genome scale to identify components of the mechanism that might be under transcriptional control.

The analysis presented here is designed to identify potential cis-regulatory elements that are controlled by events of gravitropic signal transduction. A bioinformatics approach to identify cis-regulatory elements from gravitropic microarray data is employed. The approach employed a pipeline for mining microarray and genome sequence data to identify regulatory features by searching for functional elements that are significantly over- and under-represented in the DNA regulatory regions of genes clustered based on their transcription profiles. The raw data (the expression values from a gene expression microarray experiment), analyzed here, were obtained from Kimbrough et al. [13]. Briefly, 7-day-old Arabidopsis thaliana seedlings were either rotated $135^{\circ}$ to provide a gravity stimulus or oscillated gently for $5 \mathrm{~s}$ to control for the mechanical movement of rotation. The RNA was extracted from the root tips from each group at six time points after treatment: $0,2,5,15$, 30 , and $60 \mathrm{~min}$. The RNA was amplified and hybridized to Arabidopsis ATH1 GeneChips ${ }^{\circledR}$. The resulting microarray data were analyzed, and the discovered genes were clustered based on their expression profiles. The clusters were then subjected to a motif discovery analysis pipeline to determine the interesting motifs and modules shared among the promoter regions of the genes involved in these clusters.

The remainder of the article is organized as follows: In Secton 2, the results of the microarray experiments are presented together with the analysis of the expressionbased clusters derived from said analysis. In Section 3, the methods employed during the analysis presented here are discussed in detail. Finally, in Section 4, conclusions, derived from the results and their discussion, are presented.

\section{Results}

Analysis of the microarray data (obtained from Kimbrough et al. [13]) identified 154 genes significantly up- or downregulated in the gravitropic response. The genes were grouped into 9 clusters based on similarities in their expression profiles across the time course. Promoters for all but 4 of the genes were available in a regulatory database, so a total of 150 genes, spread across the different clusters, were analyzed. For each cluster, the number of genes, and groupings of the clustered genes with similar GO Terms are shown in Table 1(a)-(i). The same process was conducted for the entire gene list (Table 1(j)). Reviewing the result, several clusters (Cluster 3, 5, and 7) share common functionality focused on the response to outside stresses. Others (Cluster 2, 6, and 8) are annotated with membrane localization. Cluster 1 is annotated as being related to signal transduction and cell communication, a feature to be expected during gravitropic response. Finally, Cluster 9 is associated with protein binding in general and heat shock protein binding in particular, which is of interest in general stress response and to be expected during gravitropic response as well. A strong relation to stress responses was strongly suggested based on the GO clusters generated for the complete gene list (Table $1(\mathrm{j})$ ).

A detailed analysis of the promoter regions of the clustered genes identified regulatory genomic signatures $[14,15]$, i.e., putative cis-regulatory elements and modules associated with gravitropic control of transcription.

Genes which have similar expression patterns typically share the same regulatory element (word) in their promoter regions. In eukaryotes, regulatory elements usually consist of $6-10$ nucleotides. Thus, for each cluster, the top five statistically over-represented 6-mers are presented in Table 2. The words, which represent putative regulatory elements, were sorted in descending order by the $S \ln (S E s)$ score ( $S$ is the number of sequences in which a word occurred and $E s$ is the number of sequences in which the word was expected to occur). The table also shows, for each word, the number of occurrences $(O)$, the reverse complement, the rank of the reverse compliment in the sorted word list, whether it is a palindrome, and the $p$-value detailing the significance of the word based on the computed number of occurrences.

Because a regulatory element may vary, a motif is often used to represent its variations. Interesting words in each gene cluster were selected for word-based clustering, wherein a motif was constructed from all words that were similar to (i.e., are within a hamming distance of 1 of) the top words. Motif logos for the top two words from each cluster are presented in Table 3.

Often, gene regulation is controlled by multiple regulatory elements (called a cis-regulatory module) that work in conjunction. To identify putative cis-regulatory modules, a module discovery algorithm was applied to the top 25 statistically over-represented words. Table 4 shows the

Table 1(a)

GO Analysis for Cluster 1

\begin{tabular}{|c|c|c|c|c|c|c|}
\hline \multicolumn{7}{|c|}{ Cluster 1 } \\
\hline Number of genes & Group & GO & Genes & Group count & Total count & Description \\
\hline 14 & 1 & GO:0007165 & at1g02340.1 & 2 & 996 & Signal transduction \\
\cline { 3 - 8 } & & GO:0007154 & at3g07890.1 & 2 & 1098 & Cell communication \\
\hline
\end{tabular}


Table 1(b)

GO Analysis for Cluster 2

\begin{tabular}{|c|c|c|c|c|c|c|}
\hline \multicolumn{7}{|c|}{ Cluster 2} \\
\hline Number of genes & Group & GO & Genes & Group count & Total count & Description \\
\hline \multirow[t]{10}{*}{18} & \multirow[t]{10}{*}{1} & GO:0005507 & at5g37990.1 & 3 & 118 & Copper ion binding \\
\hline & & GO:0016020 & at2g07695.1 & 10 & 7444 & Membrane \\
\hline & & GO:0012505 & at4g39830.1 & 7 & 4603 & Endomembrane system \\
\hline & & GO:0031225 & at3g60270.1 & 2 & 240 & Anchored to membrane \\
\hline & & & at4g11190.1 & & & \\
\hline & & & at2g33050.1 & & & \\
\hline & & & at3g16530.1 & & & \\
\hline & & & atlg78460.1 & & & \\
\hline & & & at1g79680.1 & & & \\
\hline & & & at1g70990.1 & & & \\
\hline
\end{tabular}

Table 1(c)

GO Analysis for Cluster 3

\begin{tabular}{|c|c|c|c|c|c|l|}
\hline \multicolumn{2}{|c|}{ Cluster 3 } \\
\hline Number of genes & Group & GO & Genes & Group count & Total count & \multicolumn{1}{c|}{ Description } \\
\hline \multirow{2}{*}{16} & 1 & GO:0004601 & at5g15180.1 & 2 & 120 & Peroxidase activity \\
\cline { 3 - 7 } & & GO:0016684 & at3g03670.1 & 2 & 120 & $\begin{array}{l}\text { Oxidoreductase activity, acting on } \\
\text { peroxide as acceptor }\end{array}$ \\
\cline { 3 - 7 } & & GO:0006979 & & 218 & Response to oxidative stress \\
\hline
\end{tabular}

Table $1(\mathrm{~d})$

GO Analysis for Cluster 4

\begin{tabular}{|c|c|c|c|c|c|l|}
\hline \multicolumn{7}{|c|}{ Cluster 4 (using the p value of 0.2) } \\
\hline \multirow{2}{*}{ Number of genes } & Group & GO & Genes & Group count & Total count & \multicolumn{1}{|c|}{ Description } \\
\hline \multirow{2}{*}{28} & 1 & GO:0008289 & at4g33550.1 & 2 & 163 & Lipid binding \\
\cline { 3 - 8 } & & & at5g59320.1 & & & \\
\cline { 2 - 8 } & 2 & GO:0022402 & at2g32590.1 & 2 & 185 & Cell cycle process \\
\cline { 2 - 7 } & & GO:0007049 & at3g11520.1 & 2 & 208 & Cell cycle \\
\hline
\end{tabular}

putative regulatory modules, which consist of statistically over-represented word pairs.

The selection process for the most interesting putative regulatory words (Table 5) and modules (Table 7) produced short lists. The list of significant words as putative regulatory elements and the putative elements making up the predicted modules are checked against the currently known transcription factor binding sites contained in the Arabidopsis Gene Regulatory Information Server (AGRIS) database $[16,17]$ (Tables 6 and 8 ), and TRANSFAC [18] and JASPAR [19] databases (Table 9). Among the significant words (32 in total), five of them are known and reported in AGRIS, and 14 can be found in the TRANSFAC and JASPAR databases.

Interestingly, several words are similar, i.e., they have the same core which may play an important regulatory role. Specifically, TAAGCC and TCTAAG have the same core of TAAG. TAACTC, TCTAAC, and TGTAAC not 
Table 1(e)

GO Analysis for Cluster 5

\begin{tabular}{|c|c|c|c|c|c|c|}
\hline \multicolumn{7}{|c|}{ Cluster 5} \\
\hline Number of genes & Group & $\mathrm{GO}$ & Genes & Group count & Total count & Description \\
\hline \multirow[t]{10}{*}{21} & \multirow[t]{10}{*}{1} & GO:0006869 & at5g23400.1 & 3 & 117 & Lipid transport \\
\hline & & GO:0008289 & at5g25610.1 & 3 & 163 & Lipid binding \\
\hline & & GO:0012505 & at5g53870.1 & 9 & 4603 & Endomembrane system \\
\hline & & GO:0016020 & at $4 \mathrm{~g} 12470.1$ & 10 & 7444 & Membrane \\
\hline & & GO:0006952 & at3g20820.1 & 3 & 683 & Defense response \\
\hline & & & at1g62510.1 & & & \\
\hline & & & at3g22120.1 & & & \\
\hline & & & at2g30540.1 & & & \\
\hline & & & at5g39110.1 & & & \\
\hline & & & at3g24510.1 & & & \\
\hline
\end{tabular}

Table 1 (f)

GO Analysis for Cluster 6

\begin{tabular}{|c|c|c|c|c|c|c|}
\hline \multicolumn{7}{|c|}{ Cluster 6} \\
\hline Number of genes & Group & $\mathrm{GO}$ & Genes & Group count & Total count & Description \\
\hline \multirow[t]{13}{*}{17} & \multirow[t]{3}{*}{1} & GO:0006118 & at4g37370.1 & 3 & 681 & Transport \\
\hline & & GO:0006091 & at4g31970.1 & 3 & 829 & $\begin{array}{l}\text { Generation of precursor metabolites } \\
\text { and energy }\end{array}$ \\
\hline & & GO:0019825 & at1g26380.1 & 2 & 248 & Oxygen binding \\
\hline & \multirow[t]{2}{*}{2} & GO:0005783 & at1g09080.1 & 2 & & Endoplasmic reticulum \\
\hline & & & at4g37370.1 & & & \\
\hline & \multirow[t]{8}{*}{3} & GO:0006869 & at4g36670.1 & 2 & 117 & Lipid transport \\
\hline & & GO:0012505 & at2g16005.1 & 7 & 4603 & Endomembrane system \\
\hline & & GO:0008289 & at4g31970.1 & 2 & 163 & Lipid binding \\
\hline & & GO:0016020 & at4g29020.1 & 8 & 7444 & Membrane \\
\hline & & GO:0006810 & at1g26380.1 & 4 & 1952 & Transport \\
\hline & & GO:0051234 & at5g01870.1 & 4 & 1971 & Establishment of localization \\
\hline & & GO:0051179 & at1g12090.1 & 4 & 1981 & Localization \\
\hline & & & at3g15980.1 & & & \\
\hline
\end{tabular}

only share the common core TAAC, but TAACTC and TCTAAC are also associated with Myb2 binding site motif and Myb Recognition Element (MRE) motif in Chalcone Synthase (CHS) respectively. Myb2 and MRE are known to interact [20], while Myb2 is furthermore known to be involved in the regulation of salt tolerance in Arabidopsis thaliana [21]. CCTTTC and ACCTTC share CCTT, with CCTTTC being associated with the CArG2 motif in APETALA3 (AP3). GGATAC and CAATAC share ATAC and are associated with the GATA box as well as AGATAG and AGATCA, which share AGAT, also an integral part of the GATA family. Furthermore, three pairs of words have a hamming distance of 1: CGAACC and CCAACC; TCTAAC and TGTAAC; GTATCC and GTATCT. 
Table $1(\mathrm{~g})$

GO Analysis of Cluster 7

\begin{tabular}{|c|c|c|c|c|c|c|}
\hline \multicolumn{7}{|c|}{ Cluster 7} \\
\hline Number of genes & Group & GO & Genes & Group count & Total count & Description \\
\hline \multirow[t]{27}{*}{7} & \multirow[t]{6}{*}{1} & GO:0004601 & at5g39580.1 & 3 & 120 & Peroxidase activity \\
\hline & & GO:0016684 & at1g20620.1 & 3 & 120 & $\begin{array}{l}\text { Oxidoreductase activity, acting on } \\
\text { peroxide as acceptor }\end{array}$ \\
\hline & & GO:0050832 & at2g37130.1 & 2 & 85 & Defense response to fungus \\
\hline & & GO:0009620 & at4g21850.1 & 2 & 134 & Response to fungus \\
\hline & & GO:0016491 & & 4 & 1507 & Oxidoreductase activity \\
\hline & & GO:0006952 & & 2 & 683 & Defense response \\
\hline & \multirow[t]{7}{*}{2} & GO:0008289 & at5g39580.1 & 3 & 163 & Lipid binding \\
\hline & & GO:0006869 & at4g12550.1 & 2 & 117 & Lipid transport \\
\hline & & GO:0016020 & at5g 57220.1 & 7 & 7444 & Membrane \\
\hline & & & at2g37130.1 & & & \\
\hline & & & at4g12510.1 & & & \\
\hline & & & at2g38530.1 & & & \\
\hline & & & at2g05540.1 & & & \\
\hline & \multirow[t]{3}{*}{3} & GO:0051707 & at5g39580.1 & 3 & 482 & Response to other organism \\
\hline & & GO:0009607 & at2g37130.1 & 3 & 525 & Response to biotic stimulus \\
\hline & & GO:0051704 & at4g39950.1 & 3 & 547 & Multi-organism process \\
\hline & \multirow[t]{3}{*}{4} & GO:0006091 & at5g57220.1 & 3 & 829 & $\begin{array}{l}\text { Generation of precursor metabolites } \\
\text { and energy }\end{array}$ \\
\hline & & GO:0006118 & at2g07698.1 & 2 & 681 & \\
\hline & & & at2g 46750.1 & & & \\
\hline & \multirow[t]{2}{*}{5} & GO:0019825 & at5g57220.1 & 2 & 248 & Oxygen binding \\
\hline & & & at4g39950.1 & & & \\
\hline & \multirow[t]{6}{*}{6} & GO:0012505 & at5g39580.1 & 6 & 4603 & Endomembrane system \\
\hline & & & at4g12550.1 & & & \\
\hline & & & at5g57220.1 & & & \\
\hline & & & at4g12510.1 & & & \\
\hline & & & at2g37130.1 & & & \\
\hline & & & at2g05540.1 & & & \\
\hline
\end{tabular}

\section{Methods}

\subsection{Microarray Analysis}

The raw microarray data were analyzed using Bioconductor [22], an R package suite for microarray analysis. First, the data were normalized using an un-scaled standard error (NUSE) plot for quality assessment:

$$
\operatorname{NUSE}\left(\theta_{\mathrm{gi}}\right)=\frac{\operatorname{SE}\left(\theta_{\mathrm{gi}}\right)}{\operatorname{med}_{\mathrm{i}}\left(\operatorname{SE}\left(\theta_{\text {gi }}\right)\right)}
$$

Expression values are corrected for background noise using GCRMA [23] (Fig. 1). Rank Product, a nonparametric method [24], was used to identify the differentially expressed genes in the data set. Differentially expressed genes were then selected based on the false positive prediction. A $p$-value of 0.15 was chosen and resulted in a list of 154 genes.

Once the differentially expressed genes were identified, they were clustered based on their transcriptional expression pattern. Two criteria were used to cluster the genes: 
Table 1(h)

GO Analysis of Cluster 8

\begin{tabular}{|c|c|c|c|c|c|c|}
\hline \multicolumn{7}{|c|}{ Cluster 8} \\
\hline Number of genes & Group & $\mathrm{GO}$ & Genes & Group count & Total count & Description \\
\hline \multirow[t]{13}{*}{16} & \multirow[t]{13}{*}{1} & GO:0016020 & at1g70710.1 & 12 & 7444 & Membrane \\
\hline & & GO:0031224 & at5g23840.1 & 4 & 779 & Intrinsic to membrane \\
\hline & & GO:0044425 & at3g43720.1 & 4 & 1212 & Membrane part \\
\hline & & GO:0012505 & at3g28550.1 & 7 & 4603 & Endomembrane system \\
\hline & & GO:0005623 & at1g06120.1 & 13 & 15514 & Cell \\
\hline & & GO:0044464 & at3g06460.1 & 13 & 15514 & Cell part \\
\hline & & GO:0031225 & at3g20570.1 & 2 & 240 & Anchored to membrane \\
\hline & & & at2g39510.1 & & & \\
\hline & & & at5g12940.1 & & & \\
\hline & & & at5g49770.1 & & & \\
\hline & & & at3g04320.1 & & & \\
\hline & & & at3g05020.1 & & & \\
\hline & & & at1g 47600.1 & & & \\
\hline
\end{tabular}

Table 1(i)

GO Analysis of Cluster 9

\begin{tabular}{|c|c|c|c|c|c|c|}
\hline \multicolumn{7}{|c|}{ Cluster 9 } \\
\hline Number of genes & Group & GO & Genes & Group count & Total count & Description \\
\hline 5 & 1 & GO:0031072 & at3g30450.1 & 2 & 148 & Heat shock protein binding \\
\cline { 2 - 7 } & & GO:0005515 & at2g17060.1 & 3 & 2275 & Protein binding \\
\cline { 3 - 7 } & & at2g14140.1 & & & \\
\hline
\end{tabular}

within cluster similarity and between cluster dissimilarity. The Point Accepted Mutation matrix ...[25] was used since the objective was to partition genes into several groups rather than to find the hierarchical structure. Initially, genes were clustered into 10, 15, and 20 groups. Clustering in 15 or 20 groups resulted in less than 10 genes per cluster, which is not ideal for further analysis because it does not provide large enough data sets to detect statistically interesting words. Thus, 10 clusters proved to be the most appropriate. For 10 clusters the algorithm produced an empty cluster, which is subsequently discarded.

\subsection{GO Analysis}

To provide insight into the GO terms associated with the genes of the nine clusters as well as the complete set of genes subjected to the motif discovery analysis, a GOstat [26] analysis was conducted. GOstat supported the clustering of extracted GO terms and the associated genes, allowing functional similarity assessments of the genes.

The GOstat analysis was executed for the 9 gene clusters as well as for the whole gene list containing 150 elements, since 4 of them cannot be associated with actual gene products. A $p$-value, which evaluates the matched level of genes and corresponding GO items, was set to 0.1 as a threshold. As no output can be generated for cluster 4 with the $p$-value set to 0.1 , it was to be adjusted to 0.2 .

\subsection{Statistically Over-Represented Words}

For each cluster of genes, the promoter regions were retrieved from AGRIS67. All words of the specified length 6 , which were present in the promoters, were enumerated. The expected number of occurrences for each word was computed using an order 4 Markov model (the method is described in [14]). Equations (2) and (3) show, respectively, the equations that were used to compute the expected number of occurrences and the expected number of sequences hit, for each word $w$, with $p_{w}$ being the probability of the word $w, l_{i}$ being the length of sequence $i$ (out of a total of $m$ sequences) and $v$ being the length of $w$.

$$
E o(w)=\sum_{i=1}^{m}\left(l_{i}-v+1\right) p_{w}
$$


Table 1(j)

GO Analysis of the Entire Set of 150 Genes

\begin{tabular}{|c|c|c|c|c|c|c|c|}
\hline \multirow{2}{*}{\begin{tabular}{|c|} 
Group \\
1
\end{tabular}} & \multirow{2}{*}{\begin{tabular}{|c|} 
GO \\
GO:0012505
\end{tabular}} & \multicolumn{3}{|c|}{ Genes } & \multirow{2}{*}{\begin{tabular}{c|} 
Group count \\
49
\end{tabular}} & \multirow{2}{*}{$\begin{array}{c}\text { Total count } \\
4603\end{array}$} & \multirow{2}{*}{$\begin{array}{c}\text { Description } \\
\text { Endomembrane system }\end{array}$} \\
\hline & & at1g74500.1 & at1g75780.1 & at2g33050.1 & & & \\
\hline & GO:0016020 & at3g08970.1 & at3g60270.1 & at3g15980.1 & 65 & 7444 & Membrane \\
\hline & GO:0006869 & at4g33550.1 & at3g07890.1 & at1g26380.1 & 9 & 117 & Lipid transport \\
\hline & GO:0044464 & at2g07696.1 & at5g06720.1 & at5g25610.1 & 81 & 15514 & Cell part \\
\hline & GO:0005623 & at2g38530.1 & at1g06120.1 & at1g77210.1 & 81 & 15514 & Cell \\
\hline & GO:0005507 & at1g18830.1 & at5g12940.1 & at3g16530.1 & 5 & 118 & Copper ion binding \\
\hline & GO:0031225 & at5g15180.1 & at3g04320.1 & at5g39110.1 & 6 & 240 & Anchored to membrane \\
\hline & GO:0043170 & at2g32590.1 & at3g05020.1 & at1g09080.1 & 10 & 6920 & $\begin{array}{l}\text { Macromolecule metabolic } \\
\text { process }\end{array}$ \\
\hline & GO:0005622 & at5g47990.1 & at1g47600.1 & at4g36670.1 & 18 & 9003 & Intracellular \\
\hline & GO:0050832 & at5g01870.1 & at3g 47380.1 & at3g61890.1 & 3 & 85 & Defense response to fungus \\
\hline & GO:0044424 & at4g12550.1 & at2g16005.1 & at3g03670.1 & 17 & 8514 & Intracellular part \\
\hline & GO:0043283 & at5g65600.1 & at5g26260.1 & at5g23840.1 & 6 & 4744 & Biopolymer metabolic process \\
\hline & GO:0043227 & at1g78460.1 & at5g57220.1 & at3g43720.1 & 13 & 7166 & Membrane-bounded organelle \\
\hline & GO:0043231 & at3g06460.1 & at5g11210.1 & at2g39510.1 & 13 & 7164 & $\begin{array}{l}\text { Intracellular membrane-bounded } \\
\text { organelle }\end{array}$ \\
\hline & & at5g 49770.1 & at2g05540.1 & at3g11520.1 & & & \\
\hline & & at4g29020.1 & at1g20620.1 & at5g04160.1 & & & \\
\hline & & at2g04070.1 & at5g23400.1 & at4g31970.1 & & & \\
\hline & & at3g24510.1 & at5g39580.1 & at3g22120.1 & & & \\
\hline & & at3g29970.1 & at4g39830.1 & at1g70990.1 & & & \\
\hline & & at3g20570.1 & at2g07698.1 & at1g79680.1 & & & \\
\hline & & at4g12510.1 & at2g17060.1 & at4g11190.1 & & & \\
\hline & & at5g37990.1 & at1g12090.1 & at4g12470.1 & & & \\
\hline & & at2g07695.1 & at4g17785.1 & at1g70710.1 & & & \\
\hline & & at3g20820.1 & at4g30270.1 & at1g61500.1 & & & \\
\hline & & at5g64510.1 & at5g53870.1 & at3g28550.1 & & & \\
\hline & & at4g37370.1 & at1g62510.1 & at4g28100.1 & & & \\
\hline & & at4g28710.1 & at2g37130.1 & at2g30540.1 & & & \\
\hline \multirow[t]{4}{*}{2} & GO:0008289 & at4g33550.1 & at1g12090.1 & at3g43720.1 & 11 & 163 & Lipid binding \\
\hline & & at3g 22120.1 & at5g01870.1 & at4g12550.1 & & & \\
\hline & & at4g12510.1 & at4g12470.1 & at1g62510.1 & & & \\
\hline & & at2g38530.1 & at5g59320.1 & & & & \\
\hline \multirow[t]{2}{*}{3} & GO:0006118 & at3g60270.1 & at2g07695.1 & at5g53870.1 & 13 & 681 & \\
\hline & GO:0006091 & at2g45550.1 & at1g26410.1 & at $2 \mathrm{~g} 46750.1$ & 14 & 829 & $\begin{array}{l}\text { Generation of precursor } \\
\text { metabolites and energy }\end{array}$ \\
\hline
\end{tabular}

(Continued) 
Table 1(j)

(Continued)

\begin{tabular}{|c|c|c|c|c|c|c|c|}
\hline \multirow[t]{4}{*}{ Group } & \multirow[t]{2}{*}{ GO } & \multicolumn{3}{|c|}{ Genes } & \multirow[t]{2}{*}{ Group count } & \multirow[t]{2}{*}{ Total count } & \multirow[t]{2}{*}{ Description } \\
\hline & & at4g37370.1 & at5g 47990.1 & at2g07698.1 & & & \\
\hline & & at4g31970.1 & at5g 57220.1 & at3g20570.1 & & & \\
\hline & & at2g30540.1 & at1g26380.1 & & & & \\
\hline \multirow[t]{6}{*}{4} & GO:0004601 & at1g20620.1 & at2g07695.1 & at1g64590.1 & 6 & 120 & Peroxidase activity \\
\hline & GO:0016684 & at5g39580.1 & at1g66800.1 & at5g06720.1 & 6 & 120 & $\begin{array}{l}\text { Oxidoreductase activity, acting on } \\
\text { peroxide as acceptor }\end{array}$ \\
\hline & GO:0016491 & at1g26410.1 & at5g15180.1 & at1g06120.1 & 16 & 1507 & Oxidoreductase activity \\
\hline & & at4g39830.1 & at2g37130.1 & at4g21850.1 & & & \\
\hline & & at3g03670.1 & at2g30540.1 & at1g26380.1 & & & \\
\hline & & at2g37540.1 & & & & & \\
\hline \multirow[t]{2}{*}{5} & GO:0019825 & at5g57220.1 & at4g37370.1 & at4g31970.1 & 6 & 248 & Oxygen binding \\
\hline & & at2g 45550.1 & at5g 47990.1 & at4g39950.1 & & & \\
\hline \multirow[t]{3}{*}{6} & GO:0006952 & at5g23400.1 & at2g17060.1 & at2g33050.1 & 9 & 683 & Defense response \\
\hline & & at5g39580.1 & at4g12470.1 & at2g37130.1 & & & \\
\hline & & at4g11190.1 & at3g20820.1 & at4g23670.1 & & & \\
\hline \multirow[t]{6}{*}{7} & GO:0044237 & at1g66800.1 & at1g79680.1 & at4g39950.1 & 18 & 9054 & Cellular metabolic process \\
\hline & & at1g06120.1 & at1g09080.1 & at1g01480.1 & & & \\
\hline & & at5g38020.1 & at4g11190.1 & at4g17785.1 & & & \\
\hline & & at5g65600.1 & at2g07698.1 & at1g68530.1 & & & \\
\hline & & at5g 49770.1 & at3g61890.1 & at1g61500.1 & & & \\
\hline & & at1g74500.1 & at3g08970.1 & at2g07696.1 & & & \\
\hline \multirow[t]{7}{*}{8} & GO:0044238 & at5g 49770.1 & at1g66800.1 & at2g07698.1 & 19 & 9160 & Primary metabolic process \\
\hline & & at1g79680.1 & at5g24210.1 & at3g61890.1 & & & \\
\hline & & at1g09080.1 & at1g06120.1 & at1g01480.1 & & & \\
\hline & & $\operatorname{at1g} 47600.1$ & at5g38020.1 & at4g17785.1 & & & \\
\hline & & at4g11190.1 & at5g65600.1 & at1g68530.1 & & & \\
\hline & & $\operatorname{at1g} 61500.1$ & at3g08970.1 & at2g07696.1 & & & \\
\hline & & at1g74500.1 & & & & & \\
\hline
\end{tabular}

$$
E s(w)=\sum_{j=1}^{m}\left(1-\left(1-p_{w}\right)^{l_{j}-v+1}\right)
$$

Based on these two expected values, for each word, multiple scores were computed: $\operatorname{SlnSE}$ score $\left(S \ln \left(\frac{S}{E s}\right)\right.$ [21]) and $P$-value (4),

$$
1-\sum_{j-1}^{|s|} \sum_{i=0}^{l j-v+1}\left(\begin{array}{c}
l_{j}-v+1 \\
i
\end{array}\right) p_{w}^{i}\left(1-p_{w}\right)^{l_{j}-v+1-i}
$$

A $p$-value threshold (0.05) was set for choosing sig- nificant words. Among the top five words, the top two words and the words with a $p$-value smaller than 0.05 were selected as significant.

\subsection{Word-Based Cluster}

To construct motifs, top scoring words were chosen as seeds. All enumerated words that exhibited a hamming distance of 1 from the seed word were identified and used to construct motif logos based on position weight matrices using the TFBS Perl module by Lenhard and Wassermann [27]. 
Table 2

Top 5 Statistically Over-Represented Words of the 9 Clusters

The words were sorted in descending order based on SlnSEs score

\begin{tabular}{|c|c|c|c|c|c|c|c|c|c|}
\hline \multicolumn{10}{|c|}{ Cluster 1} \\
\hline Word & $\mathrm{S}$ & Es & $\mathrm{O}$ & Eo & SlnSEs & RevComp & Position RevComp & Palindrome & Pval \\
\hline TCCCAT & 8 & 3.4851 & 8 & 4.14894 & 6.64755 & ATGGGA & 2223 & No & 0.060484 \\
\hline TGATAC & 7 & 2.96985 & 7 & 3.43636 & 6.00179 & GTATCA & 1502 & No & 0.06048 \\
\hline GGAACA & 8 & 3.83552 & 8 & 4.65882 & 5.8811 & TGTTCC & 3393 & No & 0.100222 \\
\hline CGAACC & 5 & 1.5724 & 5 & 1.69412 & 5.78417 & GGTTCG & 417 & No & 0.029237 \\
\hline TAAGCC & 6 & 2.31415 & 6 & 2.58696 & 5.7163 & GGCTTA & 1805 & No & 0.048075 \\
\hline \multicolumn{10}{|c|}{ Cluster 2} \\
\hline Word & $\mathrm{S}$ & Es & $\mathrm{O}$ & $\mathrm{E}$ & SlnSES & RevComp & Position RevComp & Palindrome & Pval \\
\hline TAACTC & 9 & 3.82579 & 10 & 4.64035 & 7.69913 & GAGTTA & 3383 & No & 0.020566 \\
\hline CCAACC & 9 & 3.92801 & 10 & 4.79208 & 7.46182 & GGTTGG & 468 & No & 0.024886 \\
\hline GGCTTA & 8 & 3.50605 & 9 & 4.17722 & 6.59961 & TAAGCC & 821 & No & 0.027108 \\
\hline GATGTA & 8 & 3.52945 & 8 & 4.21053 & 6.5464 & TACATC & 3595 & No & 0.064651 \\
\hline TCTAAG & 6 & 2.03414 & 6 & 2.2439 & 6.49013 & CTTAGA & 2799 & No & 0.027051 \\
\hline \multicolumn{10}{|c|}{ Cluster 3} \\
\hline Word & $\mathrm{S}$ & Es & $\mathrm{O}$ & $\mathrm{E}$ & SlnSES & RevComp & Position RevComp & Palindrome & Pval \\
\hline GCTCTA & 7 & 2.42874 & 7 & 2.69725 & 7.40976 & TAGAGC & 1221 & No & 0.020465 \\
\hline AGATAG & 10 & 5.36604 & 10 & 6.93243 & 6.22494 & CTATCT & 2421 & No & 0.162696 \\
\hline AGTGTT & 11 & 6.33525 & 13 & 8.68421 & 6.06942 & AACACT & 941 & No & 0.102439 \\
\hline ACCTCT & 6 & 2.21741 & 6 & 2.43902 & 5.97253 & AGAGGT & 291 & No & 0.038065 \\
\hline GCCATA & 10 & 5.63704 & 11 & 7.4 & 5.73226 & TATGGC & 2398 & No & 0.129297 \\
\hline \multicolumn{10}{|c|}{ Cluster 4} \\
\hline Word & $\mathrm{S}$ & Es & $\mathrm{O}$ & Eo & SlnSEs & RevComp & Position RevComp & Palindrome & Pval \\
\hline AGATCA & 21 & 14.3345 & 25 & 22.0914 & 8.01895 & TGATCT & 1025 & No & 0.295071 \\
\hline TCTAAC & 17 & 10.8665 & 21 & 14.6437 & 7.60798 & GTTAGA & 1510 & No & 0.068925 \\
\hline GTAAGT & 15 & 9.16075 & 18 & 11.6651 & 7.39683 & ACTTAC & 2486 & No & 0.050994 \\
\hline GTATCC & 12 & 6.50209 & 13 & 7.64925 & 7.3534 & GGATAC & 879 & No & 0.04836 \\
\hline CCATTA & 18 & 11.9867 & 21 & 16.8164 & 7.31827 & TAATGG & 3294 & No & 0.181999 \\
\hline \multicolumn{10}{|c|}{ Cluster 5} \\
\hline Word & $\mathrm{S}$ & Es & $\mathrm{O}$ & Eo & SlnSES & RevComp & Position RevComp & Palindrome & Pval \\
\hline CTCATG & 14 & 7.89675 & 20 & 10.3143 & 8.01649 & CATGAG & 1631 & No & 0.004801 \\
\hline GTATCT & 14 & 8.33286 & 16 & 11.0885 & 7.26391 & AGATAC & 3374 & No & 0.097377 \\
\hline AGAATC & 17 & 11.3066 & 26 & 17.4024 & 6.93299 & GATTCT & 3819 & No & 0.032026 \\
\hline GGATAC & 8 & 3.61972 & 9 & 4.0393 & 6.34436 & GTATCC & 3447 & No & 0.022551 \\
\hline ACTGAG & 8 & 3.65912 & 8 & 4.0885 & 6.25775 & CTCAGT & 2739 & No & 0.056571 \\
\hline
\end{tabular}

(Continued) 
Table 2

(Continued)

\begin{tabular}{|c|c|c|c|c|c|c|c|c|c|}
\hline \multicolumn{10}{|c|}{ Cluster 6} \\
\hline Word & $\mathrm{S}$ & Es & $\mathrm{O}$ & Eo & SlnSES & RevComp & Position RevComp & Palindrome & Pval \\
\hline СТСТСС & 10 & 4.95692 & 10 & 5.99394 & 7.018 & GGAGAG & 3762 & No & 0.083491 \\
\hline CAATAC & 12 & 6.98193 & 14 & 9.30677 & 6.49897 & GTATTG & 2312 & No & 0.09034 \\
\hline GCATCG & 6 & 2.04804 & 6 & 2.2029 & 6.44926 & CGATGC & NA & No & 0.025044 \\
\hline GTACGT & 10 & 5.33541 & 11 & 6.5625 & 6.2822 & ACGTAC & 959 & No & 0.070367 \\
\hline TGTAAC & 13 & 8.21936 & 19 & 11.7345 & 5.95994 & GTTACA & 3777 & No & 0.031052 \\
\hline \multicolumn{10}{|c|}{ Cluster 7} \\
\hline Word & $\mathrm{S}$ & Es & $\mathrm{O}$ & Eo & SlnSES & RevComp & Position RevComp & Palindrome & Pval \\
\hline CTTTCG & 9 & 4.32507 & 10 & 5.1831 & 6.59517 & CGAAAG & 1257 & No & 0.038953 \\
\hline ATCTGA & 11 & 6.14726 & 15 & 8.10227 & 6.40078 & TCAGAT & 1397 & No & 0.019043 \\
\hline CCATCC & 6 & 2.40417 & 6 & 2.64348 & 5.48733 & GGATGG & 2495 & No & 0.052292 \\
\hline GTGAAG & 9 & 4.92507 & 10 & 6.07981 & 5.42597 & CTTCAC & 1231 & No & 0.08951 \\
\hline TAGCTT & 13 & 8.59185 & 15 & 13.2762 & 5.38375 & AAGCTA & 3684 & No & 0.353323 \\
\hline \multicolumn{10}{|c|}{ Cluster 8} \\
\hline Word & $\mathrm{S}$ & Es & $\mathrm{O}$ & $\mathrm{E}$ & SlnSES & RevComp & Position RevComp & Palindrome & Pval \\
\hline TCATTC & 13 & 7.76988 & 17 & 11.4459 & 6.69103 & GAATGA & 128 & No & 0.073941 \\
\hline CTTAAC & 11 & 6.16998 & 13 & 8.21053 & 6.36019 & GTTAAG & 210 & No & 0.074444 \\
\hline GTGAAT & 13 & 8.03022 & 20 & 12.0482 & 6.26258 & ATTCAC & 1937 & No & 0.022049 \\
\hline ATTAAC & 14 & 8.99597 & 17 & 14.5263 & 6.19192 & GTTAAT & 2935 & No & 0.291188 \\
\hline TTACAC & 12 & 7.28713 & 16 & 10.3911 & 5.98557 & GTGTAA & 2149 & No & 0.06365 \\
\hline \multicolumn{10}{|c|}{ Cluster 9} \\
\hline Word & $\mathrm{S}$ & Es & $\mathrm{O}$ & Eo & SlnSES & RevComp & Position RevComp & Palindrome & Pval \\
\hline GAGTAT & 4 & 0.825029 & 4 & 0.913043 & 6.31452 & ATACTC & 1093 & No & 0.014108 \\
\hline GGAAGC & 4 & 0.932334 & 4 & 1.04651 & 5.82544 & GCTTCC & 2006 & No & 0.021967 \\
\hline CATCTT & 5 & 1.6255 & 6 & 2.01667 & 5.61811 & AAGATG & 2344 & No & 0.017162 \\
\hline ССТTТС & 4 & 0.982098 & 4 & 1.10976 & 5.61743 & GAAAGG & NA & No & 0.026461 \\
\hline АССТTC & 4 & 0.983157 & 4 & 1.11111 & 5.61312 & GAAGGT & 892 & No & 0.026563 \\
\hline
\end{tabular}

\subsection{Module Discovery}

To identify putative binding modules, all combinations of word pairs, and their frequencies, were enumerated. The primary statistical value that was used in module discovery was the number of sequences in which a word pair was expected to occur. This statistic is generated from the expected value of sequence hits for a single word, based on the assumption that, for each position, the probability of occurrence of each nucleotide is independent. Let $Z_{j}$ be a binary random variable, defined as follows:

$$
Z_{j}\left(W_{k}\right)= \begin{cases}1, & \text { if } W_{k} \text { occurs in sequence } j \\ 0, & \text { otherwise }\end{cases}
$$

Suppose that there are $m$ sequences, and that the length of sequence $j$ is $l_{j}$. $W_{k}$ represents a word, $\left|W_{k}\right|$ is the length of such word, and $p_{w k}$ is the probability of the specific word. Let $W=\left\{W_{1}, \ldots, W_{n}\right\}$ be the set of all words enumerated from the sequences. The number of sequences in which of a set (pair) of words is expected to occur, is computed according to (6). 
Table 3

Motif Logos

The table presents motif logos for top two over-represented words of each gene cluster. Subfigure $a$ corresponds to the highest ranked word, while Subfigure $b$ corresponds to the second highest ranked word

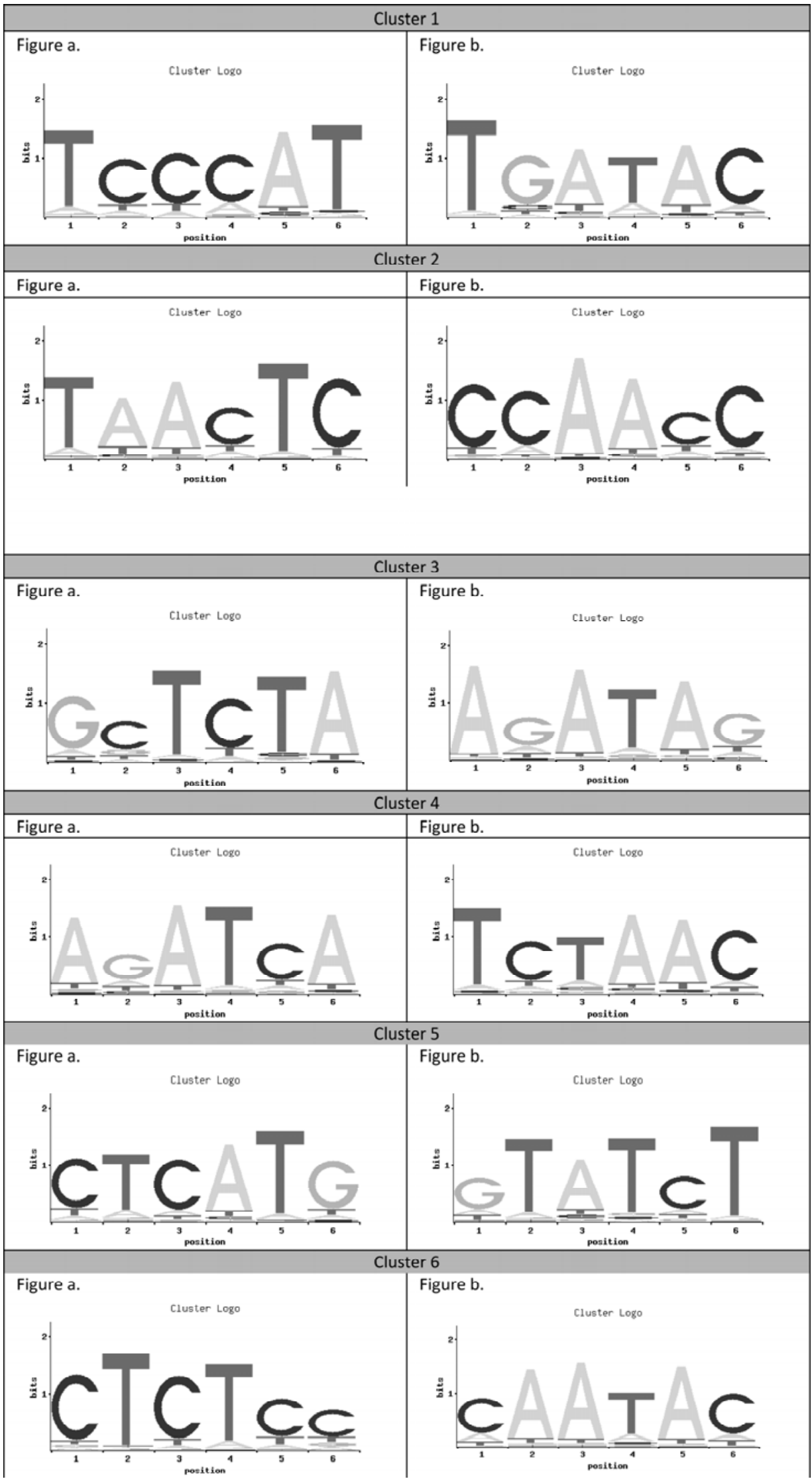

(Continued) 
Table 3

(Continued)

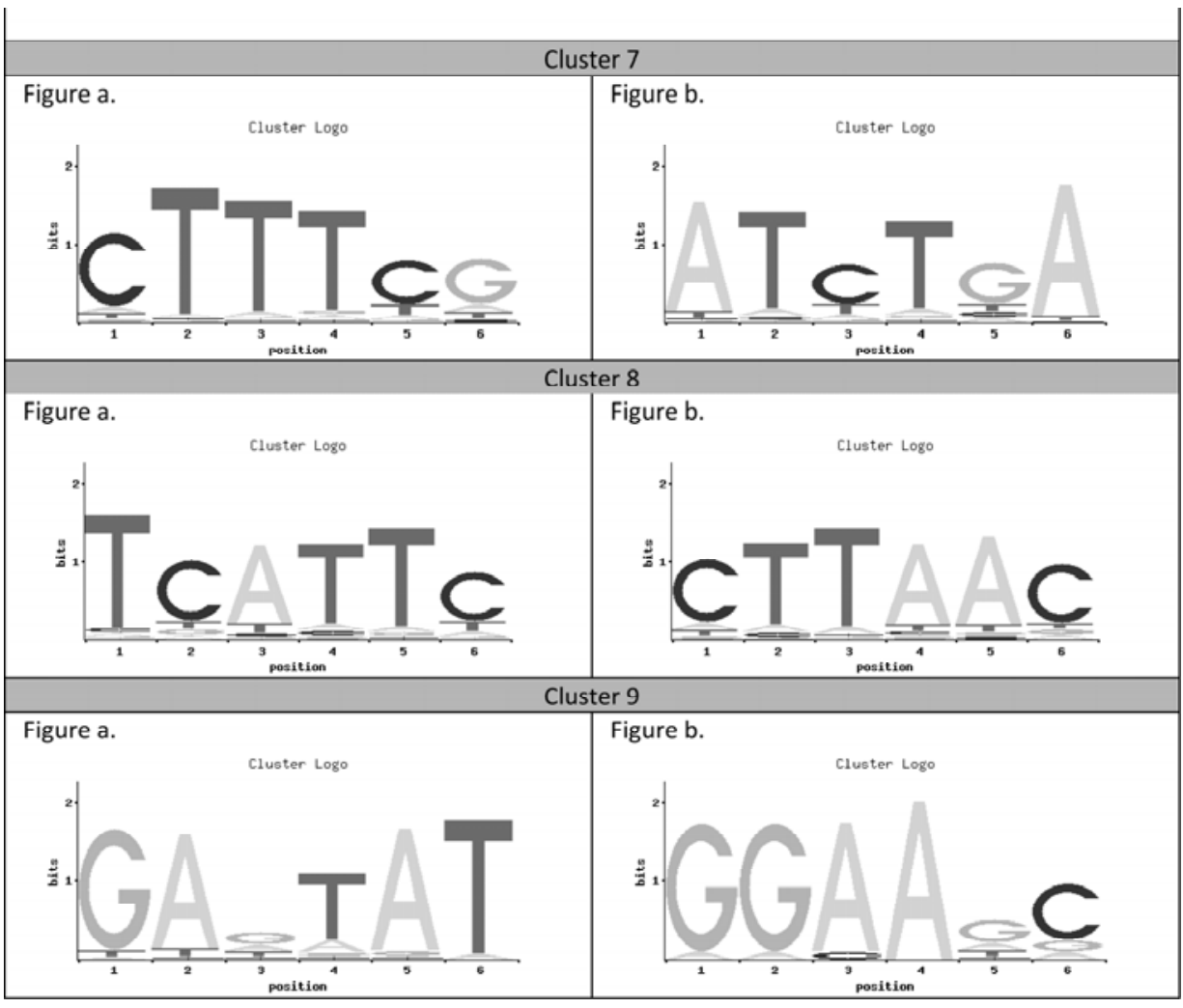

$$
E\left(\sum_{j=1}^{m} Z_{j}(W)\right)=\sum_{j=1}^{m} \prod_{k=1}^{t}\left(1-\left(1-p_{w_{k}}\right)^{l_{j}-\left|W_{k}\right|+1}\right)
$$

Besides the statistical scores, this project also computed gap and density for each word pair. The density distribution was evaluated and generated. Density for word pair is defined as $\left|W_{i}\right|+\left|W_{j}\right| /$ span, where span is the total number of nucleotides covered by the word pair and the gap between the words of the pair. Note that density is in the range of $0-100 \%$.

In this case, the top 25 statistically over-represented words of each cluster were chosen for the module discovery. All modules containing at least one word from the significant word list (Table 5) were considered as interesting modules (Table 7).

\subsection{Comparison}

Predicted cis-regulatory elements were compared with the currently available knowledge of transcription factor binding sites. The words that belong to either significant words or interesting modules were compared to the TFBS list in AGRIS. The reference information of matched motifs, including the matched binding sites and the reported publication, was reported for corresponding cis-regulatory elements.

In addition to the AGRIS-based lookup, a comparison of the word and module elements against the established transcription factor binding site knowledge compiled in the TRANSFAC8 and JASPAR9 databases was conducted using a modification of the approach by Jacox and Elnitski [28]. The sequences of each cluster were marked up with annotations regarding the TRANSFAC binding sites and subsequently analyzed for overlap with the discovered words. The matches between words and binding sites were used as the foundation to assess if a transcription factor would bind to a word by computing the ratio of actual matches between word and transcription factor binding sites and the total occurrences of the word. A threshold of 0.75 was applied to limit the results to a set of significant transcription factor matches.

\section{Conclusions}

This paper identifies regulatory genomic signatures for sets of related genes. Starting with microarray data, genomic analysis software was employed to identify putative regulatory elements and modules. Thirty two words (Table 5) were selected from the top ten over-represented words of each cluster, and are considered as putative cis-regulatory elements due to their statistical over-representation.

In addition to analyzing single words, this research project also identified significant pairs of over-represented words, which constitute putative regulatory modules. After comparing statistically over-represented modules and selected words, 55 modules, (Table 7), were chosen as putative cis-regulatory modules with the highest potential biological interest. All the modules contain a pair of words, in which at least one of them was selected from the significant word list (Table 5). Out of the 55 modules, six modules' components are both from significant words. Note that several words are shared by more than three modules. Furthermore, the average density and distance 
Table 4

Top 10 Modules

The top 10 modules for each cluster, sorted in descending order based on their $\operatorname{Sln}(\mathrm{S} / \mathrm{Es})$ score.

\begin{tabular}{|c|c|c|c|}
\hline \multicolumn{4}{|c|}{ Cluster 1} \\
\hline Module & $\mathrm{S}$ & Es & $\mathrm{S} \ln (\mathrm{S} / \mathrm{Es})$ \\
\hline $\mathrm{ACCAAT}_{-} \mathrm{GAAAAC}$ & 10 & 4.91741 & 7.09803 \\
\hline GGTTTG_ACCAAT & 8 & 3.4573 & 6.71162 \\
\hline GTCAAT_GAAAAC & 10 & 5.21406 & 6.51227 \\
\hline GACAAT_GAAAAC & 10 & 5.21406 & 6.51227 \\
\hline GTCATT_ACCAAT & 7 & 2.90888 & 6.14698 \\
\hline GTCAAT_ACCAAT & 8 & 3.91875 & 5.70935 \\
\hline ATTCCG_TGATAC & 5 & 1.77686 & 5.17295 \\
\hline ATTCCG_GTCATT & 5 & 1.77686 & 5.17295 \\
\hline TTGTGT_GAAAAC & 10 & 5.96994 & 5.15849 \\
\hline GTCAAT_GGAACA & 7 & 3.3852 & 5.08548 \\
\hline \multicolumn{4}{|c|}{ Cluster 2} \\
\hline Module & S & Es & $\mathrm{S} \ln (\mathrm{S} / \mathrm{Es})$ \\
\hline GAACTA_CCAACC & 7 & 1.30133 & 11.7777 \\
\hline ATCTTA_TTACTC & 11 & 3.9052 & 11.3915 \\
\hline TAGAAT_CCAACC & 8 & 1.95733 & 11.2629 \\
\hline CTCTAT_ACTCTA & 7 & 1.45388 & 11.0017 \\
\hline CAAATC_AATAAC & 10 & 3.48142 & 10.5514 \\
\hline TTACTC_TCTAAG & 6 & 1.03571 & 10.54 \\
\hline ATGAAG_CCAACC & 8 & 2.1875 & 10.3734 \\
\hline TTTGCA_TAACTC & 8 & 2.32562 & 9.88363 \\
\hline TTTGCA_CCAACC & 8 & 2.38558 & 9.68001 \\
\hline TAGAAT_GATGTA & 7 & 1.76565 & 9.64176 \\
\hline \multicolumn{4}{|c|}{ Cluster 3} \\
\hline Module & $\mathrm{S}$ & Es & $\mathrm{S} \ln (\mathrm{S} / \mathrm{Es})$ \\
\hline ACTGAG_GCATTG & 6 & 0.682823 & 13.0397 \\
\hline ATTAGC_GCTCTA & 6 & 0.925382 & 11.2158 \\
\hline CCCTCC_GCTCTA & 4 & 0.242921 & 11.2053 \\
\hline GCCATA_GCTCTA & 6 & 1.03879 & 10.5222 \\
\hline ACTGAG_GATAGG & 5 & 0.643251 & 10.2533 \\
\hline AGGATA_GCTCTA & 6 & 1.1675 & 9.82138 \\
\hline AAACGC_ACCTCT & 5 & 0.711836 & 9.74673 \\
\hline TGAGTT_GCTCTA & 6 & 1.18413 & 9.73652 \\
\hline ACCTAC_GCATTG & 5 & 0.718395 & 9.70087 \\
\hline CCTATA_ACTGAG & 6 & 1.20509 & 9.63123 \\
\hline
\end{tabular}

(Continued)
Table 4

(Continued)

\begin{tabular}{|c|c|c|c|}
\hline \multicolumn{4}{|c|}{ Cluster 4} \\
\hline Module & $\mathrm{S}$ & Es & $\mathrm{S} \ln (\mathrm{S} / \mathrm{Es})$ \\
\hline GCTATA_GTATCC & 10 & 2.61313 & 13.4204 \\
\hline GTAGAA_GTAAGT & 12 & 3.97595 & 13.2557 \\
\hline GTATTA_TCTTAT & 19 & 9.72642 & 12.7223 \\
\hline GTAGAA_GTATCC & 10 & 2.84172 & 12.5818 \\
\hline TGAGTC_GTAAGT & 11 & 3.55579 & 12.4225 \\
\hline GCAATG_GTATCC & 9 & 2.27587 & 12.3738 \\
\hline GAAACT_TCTTAT & 19 & 10.005 & 12.1857 \\
\hline AAGCCT_GTAAGT & 11 & 3.63476 & 12.1809 \\
\hline $\mathrm{CCACAA}_{-} \mathrm{TCTAAC}$ & 13 & 5.14182 & 12.058 \\
\hline TCTTAT_AGATCA & 18 & 9.2187 & 12.0445 \\
\hline \multicolumn{4}{|c|}{ Cluster 5} \\
\hline Module & $\mathrm{S}$ & Es & $\mathrm{S} \ln (\mathrm{S} / \mathrm{Es})$ \\
\hline GTATCT_CTCATG & 12 & 3.48888 & 14.8239 \\
\hline $\mathrm{GCTTAT}_{-} \mathrm{CTCATG}$ & 12 & 3.52175 & 14.7114 \\
\hline $\mathrm{GCTTAT}_{-} \mathrm{GTATCT}$ & 12 & 3.71202 & 14.08 \\
\hline GTTCAC_GGATAC & 8 & 1.43408 & 13.7514 \\
\hline GGATAC_CTCATG & 8 & 1.53173 & 13.2243 \\
\hline TACAAG_CTCATG & 12 & 4.2618 & 12.4226 \\
\hline AGATGT_CTCATG & 12 & 4.31969 & 12.2607 \\
\hline GTTTTC_CTCATG & 14 & 5.84617 & 12.2258 \\
\hline CAAGTG_GCTTAT & 11 & 3.78712 & 11.7292 \\
\hline GTTCAC_CTCATG & 10 & 3.0987 & 11.716 \\
\hline \multicolumn{4}{|c|}{ Cluster 6} \\
\hline Module & $\mathrm{S}$ & Es & $\mathrm{S} \ln (\mathrm{S} / \mathrm{Es})$ \\
\hline $\mathrm{AGTGAC}_{-} \mathrm{CACTCT}$ & 9 & 2.25362 & 12.4622 \\
\hline CGTGTC_CACAGT & 6 & 0.826081 & 11.8969 \\
\hline $\mathrm{CACTCT}_{-} \mathrm{CTCTCC}$ & 8 & 1.84574 & 11.7325 \\
\hline AGTGAC_CTCTCC & 8 & 1.9313 & 11.37 \\
\hline TCATAG_CAATAC & 9 & 2.55861 & 11.3198 \\
\hline GATCGA_GTACGT & 7 & 1.39758 & 11.2782 \\
\hline GGTGAT_TCATAG & 9 & 2.57787 & 11.2523 \\
\hline TGTAAC_CTCTCC & 9 & 2.60036 & 11.1742 \\
\hline $\mathrm{AACGAT}_{2}$ TGTAAC & 11 & 4.06626 & 10.9469 \\
\hline CATTTC_TGTAAC & 13 & 5.83075 & 10.4234 \\
\hline
\end{tabular}

(Continued) 
Table 4

(Continued)

\begin{tabular}{|c|c|c|c|}
\hline \multicolumn{4}{|c|}{ Cluster 7} \\
\hline Module & $\mathrm{S}$ & Es & $\mathrm{S} \ln (\mathrm{S} / \mathrm{Es})$ \\
\hline ATCACA_CTTTCG & 9 & 2.48752 & 11.5734 \\
\hline CGGGTA_TGCCAG & 5 & 0.495852 & 11.5546 \\
\hline TAGCTT_CTTTCG & 9 & 2.61704 & 11.1166 \\
\hline CAACGT_ATCTGA & 9 & 2.77125 & 10.6013 \\
\hline CTTAAG_ATCTGA & 9 & 2.78466 & 10.5579 \\
\hline ATCACA_ATCTGA & 10 & 3.52527 & 10.4263 \\
\hline GTTCTC_GTCTAA & 6 & 1.07328 & 10.3263 \\
\hline GTAACC_ATCTGA & 7 & 1.60119 & 10.3261 \\
\hline GTCTAA_ATCTGA & 8 & 2.23224 & 10.2115 \\
\hline \multicolumn{4}{|c|}{ Cluster 8} \\
\hline Module & $\mathrm{S}$ & Es & $\mathrm{S} \ln (\mathrm{S} / \mathrm{Es})$ \\
\hline GTGAAT_TCATTC & 12 & 4.38729 & 12.0743 \\
\hline ATTAAC_CTTAAC & 11 & 3.90788 & 11.3839 \\
\hline TTGCTG_ATTAAC & 12 & 4.69791 & 11.2535 \\
\hline TCGAAC_GTCAAG & 8 & 1.96711 & 11.223 \\
\hline ACATTG_TTACAC & 11 & 4.0788 & 10.913 \\
\hline CCCATG_ATAGTG & 7 & 1.49161 & 10.8224 \\
\hline TTACAC_GTGAAT & 11 & 4.12317 & 10.794 \\
\hline ACATTG_CTTAAC & 10 & 3.4685 & 10.5886 \\
\hline TCGAAC_TTGCTG & 9 & 2.80362 & 10.4968 \\
\hline GTGAAT_CTTAAC & 10 & 3.50615 & 10.4807 \\
\hline \multicolumn{4}{|c|}{ Cluster 9} \\
\hline Module & $\mathrm{S}$ & Es & $\mathrm{S} \ln (\mathrm{S} / \mathrm{Es})$ \\
\hline ACCTTC_GAGTAT & 4 & 0.178573 & 12.4362 \\
\hline CACCGA_GGAAGC & 4 & 0.215494 & 11.6845 \\
\hline CAACTC_CCTTTC & 4 & 0.246191 & 11.1518 \\
\hline CCTCAC_GAGTAT & 4 & 0.280597 & 10.6285 \\
\hline CATCTT_GAGTAT & 4 & 0.292942 & 10.4563 \\
\hline CTGACA_GAGTAT & 4 & 0.313277 & 10.1879 \\
\hline CATCTT_GGAAGC & 4 & 0.330686 & 9.97153 \\
\hline CTATGT_GGAAGC & 4 & 0.33226 & 9.95253 \\
\hline CCTCAC_ACCTTC & 4 & 0.333838 & 9.93358 \\
\hline CGAATC_CCTTTC & 4 & 0.335205 & 9.91723 \\
\hline
\end{tabular}

Table 5

Significant Words

Selected over-represented words from each cluster's top five over-represented words. For every cluster, the top two over-represented words (as determined by the SlnSEs score) were chosen, in addition to words with $p$-values less than 0.05 .

\begin{tabular}{|c|c|c|}
\hline \multicolumn{3}{|c|}{ Cluster 1} \\
\hline Word & SlnSEs & $p$-Value \\
\hline TCCCAT & 6.64755 & 0.060484 \\
\hline TGATAC & 6.00179 & 0.06048 \\
\hline CGAACC & 5.078417 & 0.029237 \\
\hline TAAGCC & 5.07163 & 0.048075 \\
\hline \multicolumn{3}{|c|}{ Cluster 2} \\
\hline Word & SlnSEs & $p$-Value \\
\hline TAACTC & 7.69913 & 0.020566 \\
\hline CCAACC & 7.46182 & 0.024886 \\
\hline GGCTTA & 6.59961 & 0.027108 \\
\hline TCTAAG & 6.049013 & 0.027051 \\
\hline \multicolumn{3}{|c|}{ Cluster 3} \\
\hline Word & SlnSEs & $p$-Value \\
\hline GCTCTA & 7.40976 & 0.020465 \\
\hline AGATAG & 6.22494 & 0.162696 \\
\hline ACCTCT & 5.97253 & 0.038065 \\
\hline \multicolumn{3}{|c|}{ Cluster 4} \\
\hline Word & SlnSEs & $p$-Value \\
\hline AGATCA & 8.01895 & 0.295071 \\
\hline TCTAAC & 7.60798 & 0.068925 \\
\hline GTATCC & 7.3534 & 0.04836 \\
\hline \multicolumn{3}{|c|}{ Cluster 5} \\
\hline Word & SlnSEs & $p$-Value \\
\hline CTCATG & 8.01649 & 0.004801 \\
\hline GTATCT & 7.26391 & 0.097377 \\
\hline AGAATC & 6.93299 & 0.032026 \\
\hline GGATAC & 6.34436 & 0.022551 \\
\hline \multicolumn{3}{|c|}{ Cluster 6} \\
\hline Word & SlnSEs & $p$-Value \\
\hline СТСТСС & 7.018 & 0.083491 \\
\hline CAATAC & 6.49897 & 0.09034 \\
\hline GCATCG & 6.44926 & 0.025044 \\
\hline TGTAAC & 5.95994 & 0.031052 \\
\hline \multicolumn{3}{|c|}{ Cluster 7} \\
\hline Word & SlnSEs & $p$-Value \\
\hline CTTTCG & 6.59517 & 0.038953 \\
\hline ATCTGA & 6.40078 & 0.019043 \\
\hline
\end{tabular}

(Continued) 
Table 5

(Continued)

\begin{tabular}{|c|c|c|}
\hline \multicolumn{3}{|c|}{ Cluster 8} \\
\hline Word & SlnSEs & P-Value \\
\hline TCATTC & 6.69103 & 0.073941 \\
\hline CTTAAC & 6.36019 & 0.074444 \\
\hline GTGAAT & 6.36019 & 0.022049 \\
\hline \multicolumn{3}{|c}{ Cluster 9} \\
\hline Word & SlnSEs & P-Value \\
\hline GAGTAT & 6.31452 & 0.014108 \\
\hline GGAAGC & 5.82544 & 0.021967 \\
\hline CATCTT & 5.61811 & 0.017162 \\
\hline CCTTTC & 5.61743 & 0.026461 \\
\hline ACCTTC & 5.61312 & 0.026563 \\
\hline
\end{tabular}

are associated with each module (Table 7). Note that, while most modules have the density lower than $10 \%$, module CCTCAC_GAGTAT has a density of $39.2106 \%$, with an average distance of 179 bps between its elements.

\section{Acknowledgements}

We would like to acknowledge Frank Drews, Matt Wiley, Rami Al-Ouran, Lee Nau, and Kyle Kurz for their support in the development of the software applied in this research. We acknowledge the support of the Ohio University Stocker Endowment, Ohio University's Graduate Research and Education Board (GERB), the Ohio Supercomputer Center, and the Choose Ohio First Initiative of the University System of Ohio. Additionally, salaries and research support were provided by state funds appropriated to the Ohio Plant Biotechnology Consortium through The Ohio State University, Ohio Agricultural Research and Development Center.

Table 6

AGRIS Look-up of Significant Words

This table reports the words for the specific clusters that are contained in the AGRIS database

\begin{tabular}{|c|c|c|c|c|c|}
\hline \multicolumn{6}{|c|}{ Cluster 1} \\
\hline \multicolumn{6}{|c|}{-} \\
\hline \multicolumn{6}{|c|}{ Cluster 2} \\
\hline Word & SlnSEs & $P$-Value & Name & Consensus Motif & Reference \\
\hline TAACTC & 7.69913 & 0.020566 & MYB2 binding site motif & TAACT(G/C)GTT & 29 \\
\hline \multicolumn{6}{|c|}{ Cluster 3} \\
\hline Word & SlnSEs & $P$-Value & Name & Consensus Motif & Reference \\
\hline AGATAG & 6.22494 & 0.162696 & GATA promoter motif & {$[\mathrm{AT}] \mathrm{GATA}[\mathrm{GA}]$} & 30 \\
\hline \multicolumn{6}{|c|}{ Cluster 4} \\
\hline Word & SlnSEs & $P$-Value & Name & Consensus Motif & Reference \\
\hline TCTAAC & 7.60798 & 0.068925 & MRE motif in CHS & TCTAACCTACCA & 31 \\
\hline \multicolumn{6}{|c|}{ Cluster 5} \\
\hline Word & SlnSEs & $P$-Value & Name & Consensus Motif & Reference \\
\hline \multirow[t]{2}{*}{ GTATCT } & 7.26391 & 0.097377 & $\begin{array}{l}\text { EIL1 BS in ERF1; } \\
\text { EIL2 BS in ERF1; } \\
\text { EIL3 BS in ERF1 }\end{array}$ & TTCAAGGGGGCATGTATCTTGAA & 32 \\
\hline & & & EIN3 BS in ERF1 & GGATTCAAGGGGGCATGTATCTTGAATCC & \\
\hline \multicolumn{6}{|c|}{ Cluster 6} \\
\hline \multicolumn{6}{|c|}{-} \\
\hline \multicolumn{6}{|c|}{ Cluster 7} \\
\hline \multicolumn{6}{|c|}{-} \\
\hline \multicolumn{6}{|c|}{ Cluster 8} \\
\hline \multicolumn{6}{|c|}{-} \\
\hline \multicolumn{6}{|c|}{ Cluster 9} \\
\hline Word & SlnSEs & $p$-Value & Name & Consensus Motif & Reference \\
\hline ССТTTC & 5.61743 & 0.026461 & CArG2 motif in AP3 & CTTACCTTTCATGGATTA & 33 \\
\hline
\end{tabular}


Table 7

Significant Modules

This table presents interesting modules selected from top 10 over-represented modules of each cluster. For each module, the statistical value, SlnSE score, and several features, including density, and distance are shown in this table

\begin{tabular}{|c|c|c|c|c|}
\hline \multicolumn{5}{|c|}{ Cluster 1} \\
\hline Module & SlnSEs & Significant Word Contained & Average Density & Average Distance \\
\hline ATTCCG_TGATAC & 5.17295 & TGATAC & $2.22 \%$ & 403 \\
\hline \multicolumn{5}{|c|}{ Cluster 2} \\
\hline Module & SlnSEs & Significant Word Contained & Average Density & Average Distance \\
\hline GAACTA_CCAACC & 11.7777 & CCAACC & $2.04 \%$ & 895 \\
\hline TAGAAT_CCAACC & 11.2629 & CCAACC & $5.59 \%$ & 422 \\
\hline TTACTC_TCTAAG & 10.54 & TCTAAG & $22.72 \%$ & 549 \\
\hline ATGAAG_CCAACC & 10.3734 & CCAACC & $6.11 \%$ & 316 \\
\hline TTTGCA_TAACTC & 9.88363 & TAACTC & $7.27 \%$ & 631 \\
\hline TTTGCA_CCAACC & 9.68001 & CCAACC & $2.62 \%$ & 753 \\
\hline \multicolumn{5}{|c|}{ Cluster 3} \\
\hline Module & SlnSEs & Significant Word Contained & Average Density & Average Distance \\
\hline ATTAGC_GCTCTA & 11.2158 & GCTCTA & $8.70 \%$ & 518 \\
\hline CCCTCC_GCTCTA & 11.2053 & GCTCTA & $15.40 \%$ & 893 \\
\hline GCCATA_GCTCTA & 10.5222 & GCTCTA & $2.56 \%$ & 552 \\
\hline AGGATA_GCTCTA & 9.82138 & GCTCTA & $7.72 \%$ & 909 \\
\hline AAACGC_ACCTCT & 9.74673 & ACCTCT & $7.16 \%$ & 891 \\
\hline TGAGTT_GCTCTA & 9.73652 & GCTCTA & $4.91 \%$ & 673 \\
\hline \multicolumn{5}{|c|}{ Cluster 4} \\
\hline Module & SlnSEs & Significant Word Contained & Average Density & Average Distance \\
\hline GCTATA_GTATCC & 13.4204 & GTATCC & $6.10 \%$ & 715 \\
\hline GTAGAA_GTATCC & 12.5818 & GTATCC & $2.18 \%$ & 1047 \\
\hline GCAATG_GTATCC & 12.3738 & GTATCC & $2.13 \%$ & 916 \\
\hline CCACAA_TCTAAC & 12.058 & TCTAAC & $10.55 \%$ & 463 \\
\hline TCTTAT_AGATCA & 12.0445 & GTATCT; AGATCA & $2.58 \%$ & 739 \\
\hline \multicolumn{5}{|c|}{ Cluster 5} \\
\hline Module & SlnSEs & Significant Word Contained & Average Density & Average Distance \\
\hline GTATCT_CTCATG & 14.7114 & CTCATG & $21.51 \%$ & 543 \\
\hline GCTTAT_GTATCT & 14.08 & GTATCT & $6.84 \%$ & 670 \\
\hline GTTCAC_GGATAC & 13.7514 & GGATAC & $2.41 \%$ & 913 \\
\hline GGATAC_CTCATG & 13.2243 & CTCATG & $6.46 \%$ & 933 \\
\hline TACAAG_CTCATG & 12.4226 & CTCATG & $3.15 \%$ & 905 \\
\hline AGATGT_CTCATG & 12.2607 & CTCATG & $2.90 \%$ & 728 \\
\hline GTTTTC_CTCATG & 12.2258 & CTCATG & $3.96 \%$ & 704 \\
\hline GTTCAC_CTCATG & 11.716 & CTCATG & $3.13 \%$ & 727 \\
\hline
\end{tabular}


Table 7

(Continued)

\begin{tabular}{|c|c|c|c|c|}
\hline \multicolumn{5}{|c|}{ Cluster 6} \\
\hline Module & SlnSEs & Significant Word Contained & Average Density & Average Distance \\
\hline CACTCT_CTCTCC & 11.7325 & СТCTCC & $17.47 \%$ & 807 \\
\hline AGTGAC_CTCTCC & 11.37 & СТCTCC & $3.84 \%$ & 981 \\
\hline TCATAG_CAATAC & 11.3198 & CAATAC & $1.87 \%$ & 1045 \\
\hline TGTAAC_CTCTCC & 11.1742 & TGTAAC; CTCTCC & $1.92 \%$ & 926 \\
\hline AACGAT_TGTAAC & 10.9469 & TGTAAC & $1.43 \%$ & 1091 \\
\hline CATTTC_TGTAAC & 10.4234 & TGTAAC & $3.16 \%$ & 862 \\
\hline \multicolumn{5}{|c|}{ Cluster 7} \\
\hline Module & SlnSEs & Significant Word Contained & Average Density & Average Distance \\
\hline ATCACA_CTTTCG & 11.5734 & CTTTCG & $3.70 \%$ & 960 \\
\hline TAGCTT_CTTTCG & 11.1166 & CTTTCG & $16.84 \%$ & 1118 \\
\hline CAACGT_ATCTGA & 10.6013 & ATCTGA & $2.80 \%$ & 738 \\
\hline CTTAAG_ATCTGA & 10.5579 & ATCTGA & $6.86 \%$ & 559 \\
\hline ATCACA_ATCTGA & 10.4263 & ATCTGA & $8.24 \%$ & 545 \\
\hline GTAACC_ATCTGA & 10.3261 & ATCTGA & $1.94 \%$ & 1060 \\
\hline GTCTAA_ATCTGA & 10.2115 & ATCTGA & $3.06 \%$ & 788 \\
\hline \multicolumn{5}{|c|}{ Cluster 8} \\
\hline Module & SlnSEs & Significant Word Contained & Average Density & Average Distance \\
\hline GTGAAT_TCATTC & 12.0743 & GTGAAT; TCATTC & $2.93 \%$ & 752 \\
\hline ATTAAC_CTTAAC & 11.3839 & CTTAAC & $3.14 \%$ & 780 \\
\hline TTACAC_GTGAAT & 10.794 & GTGAAT & $19.27 \%$ & 403 \\
\hline ACATTG_CTTAAC & 10.5886 & CTTAAC & $3.78 \%$ & 507 \\
\hline GTGAAT_CTTAAC & 10.4807 & GTGAAT; CTTAAC & $6.08 \%$ & 702 \\
\hline \multicolumn{5}{|c|}{ Cluster 9} \\
\hline Module & SlnSEs & Significant Word Contained & Average Density & Average Distance \\
\hline ACCTTC_GAGTAT & 12.4362 & GAGTAT & $1.81 \%$ & 682 \\
\hline CACCGA_GGAAGC & 11.6845 & GGAAGC & $1.59 \%$ & 806 \\
\hline CAACTC_CCTTTC & 11.1518 & CCTTTC & $1.85 \%$ & 828 \\
\hline CCTCAC_GAGTAT & 10.6285 & GAGTAT & $39.21 \%$ & 179 \\
\hline CATCTT_GAGTAT & 10.4563 & CATCTT; GAGTAT & $2.84 \%$ & 655 \\
\hline CTGACA_GAGTAT & 10.1879 & GAGTAT & $1.53 \%$ & 835 \\
\hline CATCTT_GGAAGC & 9.97153 & CATCTT; GGAAGC & $2.60 \%$ & 621 \\
\hline CTATGT_GGAAGC & 9.95253 & GGAAGC & $0.70 \%$ & 1758 \\
\hline CCTCAC_ACCTTC & 9.93358 & ACCTTC & $2.24 \%$ & 977 \\
\hline CGAATC_CCTTTC & 9.91723 & СCTTTC & $2.19 \%$ & 613 \\
\hline
\end{tabular}


Table 8

AGRIS Look-up of Interesting Modules

The information from the AGRIS database 67 for the words in module's component is provided

\begin{tabular}{|c|c|c|c|c|c|}
\hline \multicolumn{6}{|c|}{ Cluster 1} \\
\hline \multicolumn{6}{|c|}{-} \\
\hline \multicolumn{6}{|c|}{ Cluster 2} \\
\hline Word & SlnSEs & P-Value & Name & Consensus Motif & Reference \\
\hline TAACTC & 7.69913 & 0.020566 & MYB2 binding site motif & $\mathrm{TAACT}(\mathrm{G} / \mathrm{C}) \mathrm{GTT}$ & 29 \\
\hline \multicolumn{6}{|c|}{ Cluster 3} \\
\hline Word & SlnSEs & P-Value & Name & Consensus Motif & Reference \\
\hline AGATAG & 6.22494 & 0.162696 & GATA promoter motif & {$[\mathrm{AT}] \mathrm{GATA}[\mathrm{GA}]$} & 30 \\
\hline \multicolumn{6}{|c|}{ Cluster 4} \\
\hline Word & SlnSEs & P-Value & Name & Consensus Motif & Reference \\
\hline TCTAAC & 7.60798 & 0.068925 & MRE motif in CHS & TCTAACCTACCA & 31 \\
\hline \multicolumn{6}{|c|}{ Cluster 5} \\
\hline Word & SlnSEs & P-Value & Name & Consensus Motif & Reference \\
\hline \multirow[t]{2}{*}{ GTATCT } & 7.26391 & 0.097377 & $\begin{array}{l}\text { EIL1 BS in ERF1; } \\
\text { EIL2 BS in ERF1; } \\
\text { EIL3 BS in ERF1 }\end{array}$ & TTCAAGGGGGCATGTATCTTGAA & 32 \\
\hline & & & EIN3 BS in ERF1 & GGATTCAAGGGGGCATGTATCTTGAATCC & \\
\hline \multicolumn{6}{|c|}{ Cluster 6} \\
\hline Word & SlnSEs & P-Value & Name & Consensus Motif & Reference \\
\hline TCATAG & 11.3198 & - & LS5 promoter motif & ACGTCATAGA & 34 \\
\hline \multicolumn{6}{|c|}{ Cluster 7} \\
\hline \multicolumn{6}{|c|}{-} \\
\hline \multicolumn{6}{|c|}{ Cluster 8} \\
\hline \multicolumn{6}{|c|}{-} \\
\hline \multicolumn{6}{|c|}{ Cluster 9} \\
\hline Word & SlnSEs & P-Value & Name & Consensus Motif & Reference \\
\hline ССТTTC & 5.61743 & 0.026461 & CArG2 motif in AP3 & CTTACCTTTCATGGATTA & 33 \\
\hline
\end{tabular}


Table 9

TRANSFAC Analysis

For each significant word, this table shows transcription factors known to be bind to the word, as published in TRANSFAC8 and JASPAR 9 database. Any known binding sites that hit significant words more than $3 / 4$ of their total occurrences are shown

\begin{tabular}{|c|c|c|c|c|c|c|c|}
\hline \multicolumn{8}{|c|}{ Cluster 1} \\
\hline Word & $\mathrm{S}$ & $\mathrm{O}$ & TF_ID & TF_Name & Score_Range & TFBS & Matches \\
\hline TGATAC & 7 & 7 & MA0035 & Gata1 & $90.93-90.93$ & NGATNN & $14 \backslash 14$ \\
\hline TGATAC & 7 & 7 & MA0037 & Gata3 & $86.31-86.31$ & HGATWR & $14 \backslash 14$ \\
\hline \multicolumn{8}{|c|}{ Cluster 2} \\
\hline \multicolumn{8}{|c|}{-} \\
\hline \multicolumn{8}{|c|}{ Cluster 3} \\
\hline Word & $\mathrm{S}$ & $\mathrm{O}$ & TF_ID & TF_Name & Score_Range & TFBS & Matches \\
\hline AGATAG & 10 & 10 & MA0035 & Gata1 & $94.35-94.35$ & NGATNN & $18 \backslash 18$ \\
\hline AGATAG & 10 & 10 & MA0037 & Gata3 & $100.00-100.00$ & HGATWR & $18 \backslash 18$ \\
\hline AGATAG & 10 & 10 & V\$GATA3_01 & Gata3 & $85.26-95.56$ & NNGATWDNN & $16 \backslash 18$ \\
\hline AGATAG & 10 & 10 & V\$GATA6_01 & Gata6 & $85.51-92.33$ & NNHGATWNNN & $18 \backslash 18$ \\
\hline AGATAG & 10 & 10 & V\$GATA_Q6 & Gata & $91.43-98.09$ & WGATARN & $18 \backslash 18$ \\
\hline \multicolumn{8}{|c|}{ Cluster 4} \\
\hline Word & $\mathrm{S}$ & $\mathrm{O}$ & TF_ID & TF_Name & Score_Range & TFBS & Matches \\
\hline AGATCA & 21 & 25 & $\mathrm{~V} \$ \mathrm{HNF} 4$ Q66_02 & HNF4 & $88.87-88.87$ & AGKYCA & $48 \backslash 48$ \\
\hline AGATCA & 21 & 25 & $\mathrm{~V} \$ \mathrm{HNF} 4$ Q66_03 & $\mathrm{HNF} 4$ & $90.67-90.67$ & NGDBCA & $48 \backslash 48$ \\
\hline GTATCC & 12 & 13 & MA0035 & Gata1 & $93.07-93.07$ & NGATNN & $19 \backslash 19$ \\
\hline \multicolumn{8}{|c|}{ Cluster 5} \\
\hline Word & $\mathrm{S}$ & $\mathrm{O}$ & TF_ID & TF_Name & Score_Range & TFBS & Matches \\
\hline CTCATG & 14 & 20 & MA0089 & TCF11-MafG & $87.05-87.05$ & NATGAC & $29 \backslash 29$ \\
\hline GTATCT & 14 & 16 & MA0035 & Gata1 & $92.07-92.07$ & NGATNN & $26 \backslash 26$ \\
\hline GTATCT & 14 & 16 & MA0037 & Gata3 & $87.55-87.55$ & HGATWR & $26 \backslash 26$ \\
\hline AGAATC & 17 & 26 & V\$STAT5A_04 & STAT5A & $85.03-88.96$ & NNNTTCYN & $32 \backslash 38$ \\
\hline GGATAC & 8 & 9 & MA0035 & Gata1 & $93.07-93.07$ & NGATNN & $11 \backslash 11$ \\
\hline \multicolumn{8}{|c|}{ Cluster 6} \\
\hline Word & $\mathrm{S}$ & $\mathrm{O}$ & TF_ID & TF_Name & Score_Range & TFBS & Matches \\
\hline GCATCG & 6 & 6 & MA0035 & Gata1 & $97.24-97.24$ & NGATNN & $6 \backslash 6$ \\
\hline \multicolumn{8}{|c|}{ Cluster 7} \\
\hline Word & $\mathrm{S}$ & $\mathrm{O}$ & TF_ID & TF_Name & Score_Range & TFBS & Matches \\
\hline CTTTCG & 9 & 10 & MA0080 & SPI1 & $85.47-85.47$ & VGGAAS & $21 \backslash 21$ \\
\hline ATCTGA & 11 & 15 & $\mathrm{~V} \$ \mathrm{CAP} \_01$ & CAP & $85.57-94.08$ & NCABHNNN & $25 \backslash 25$ \\
\hline
\end{tabular}

(Continued) 
Table 9

(Continued)

\begin{tabular}{|c|c|c|c|c|c|c|c|}
\hline \multicolumn{7}{|c|}{ Cluster 8} \\
\hline Word & S & O & TF_ID & TF_Name & Score_Range & TFBS & Matches \\
\hline TCATTC & 13 & 17 & V\$CAP_01 & CAP & $89.34-97.86$ & NCABHNNN & $31 \backslash 31$ \\
\hline TCATTC & 13 & 17 & V\$GEN_INI2_B & GEN INI2 & $85.83-100.00$ & BBNCANTB & $24 \backslash 31$ \\
\hline TCATTC & 13 & 17 & V\$GEN_INI_B & GEN INI & $86.69-100.00$ & NBNCANTB & $24 \backslash 31$ \\
\hline \multicolumn{7}{|c|}{ Cluster 9 } \\
\hline Word & S & O & TF_ID & TF_Name & Score_Range & TFBS & Matches \\
\hline CATCTT & 5 & 6 & V\$CAP_01 & CAP & $86.97-93.14$ & NCABHNNN & $10 \backslash 12$ \\
\hline GGAAGC & 4 & 4 & V\$CETS168_Q6 & CETS168 & $85.92-100.00$ & CMGGAAGY & $6 \backslash 7$ \\
\hline GGAAGC & 4 & 4 & V\$PEA3_Q6 & PEA3 & $89.16-91.24$ & ACWTCCK & $6 \backslash 7$ \\
\hline GGAAGC & 4 & 4 & V\$STAT3_02 & STAT3 & $91.22-98.25$ & NNNTTCCN & $7 \backslash 7$ \\
\hline
\end{tabular}

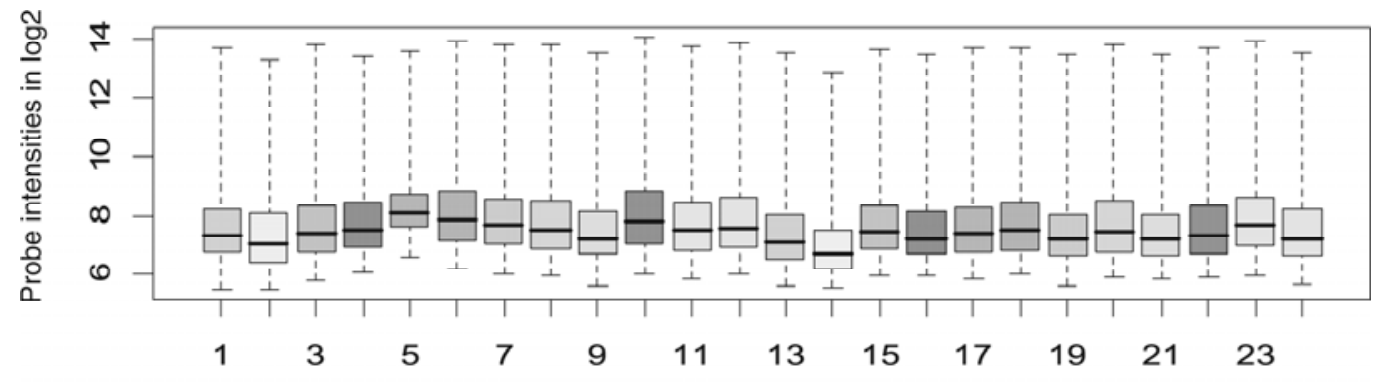

Sample number

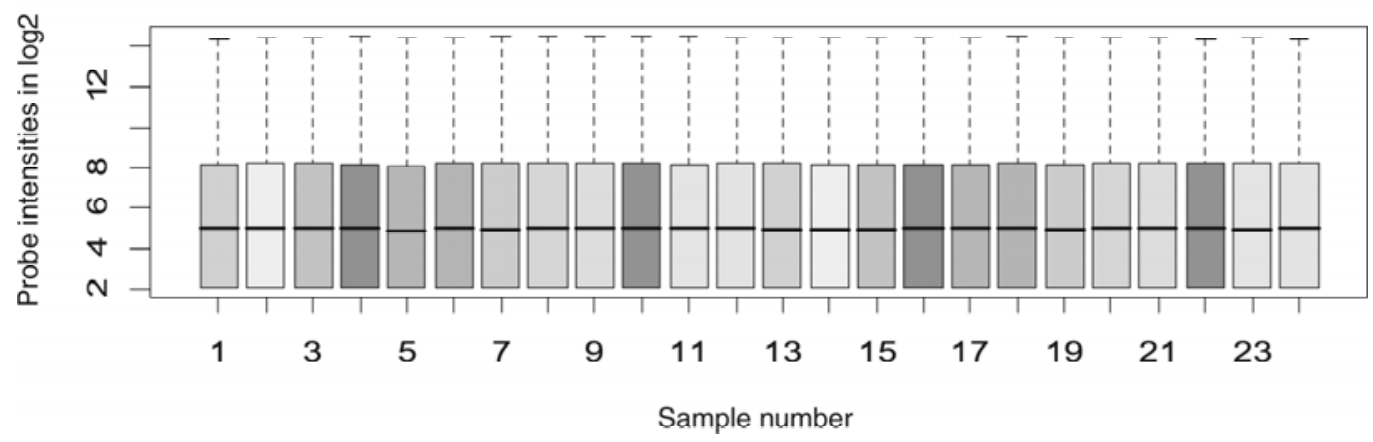

Figure 1. The normalization of samples. The raw data (top) before normalization and the data after GCRMA normalization (bottom) are compared.

\section{References}

[1] F.D. Sack, Plant gravity sensing International Review of Cytology-a Survey of Cell Biology, 127, 1991, 193-252.

[2] J.Z. Kiss, Mechanisms of the early phases of plant gravitropism, CRC Critical Review Plant Science, 19(6), 2000, 551-573.

[3] T. Caspar \& B.G. Pickard, Gravitropism in a starchless mutant of Arabidopsis - Implications for the Starch-Statolith Theory of Gravity Sensing, Planta, 17r(2), 1989, 185-197.

[4] F.D. Sack \& J.Z. Kiss, Rootcap structure in wild-type and in a starchless Mutant of Arabidopsis, American Journal of Botany, 76(2), 1989, 454-464.

[5] B.R. Harrison \& P.H. Masson, ARL2, ARG1 and PIN3 define a gravity signal transduction pathway in root statocytes, Plant Journal, 53(2), 2008, 380-392.
[6] A.C. Scott \& N.S. Allen, Changes in cytosolic pH within Arabidopsis root columella cells play a key role in the early signaling pathway for root gravitropism, Plant Physiol, 121(4), 1999, 1291-1298.

[7] E.B. Blancaflor \& P.H. Masson, Plant gravitropism: Unraveling the ups and downs of a complex process, Plant Physiology, 133(4), 2003, 1677-1690.

[8] I.Y. Perera, I. Heilmann, \& W.F. Boss, Transient and sustained increases in inositol 1,4,5-trisphosphate precede the differential growth response in gravistimulated maize pulvini. Proceedings National Academy Sciences United States of America, 96(10), 1999, 5838-5843.

[9] W.F. Boss, I.Y. Perera, J. Love, \& I. Heilmann, Altering phosphoinositide metabolism by expressing human type I inos- 
itol polyphosphate 5' phosphatise in tobacco cells. Molecular Biology of the Cell, 12: 2001, 820.

[10] J.H. Joo, Y.S. Bae, \& J.S. Lee, Role of auxin-induced reactive oxygen species in root gravitropism, Plant Physiology, 126(3), 2001, 1055-1060.

[11] A.M. Clore, S.M. Doore, \& S.M.N. Tinnirello, Increased levels of reactive oxygen species and expression of a cytoplasmic aconitase/iron regulatory protein 1 homolog during the early response of maize pulvini to gravistimulation, Plant Cell and Environment, 31(1), 2008, 144-158.

[12] P.B. Kaufman, L.-L. Wu, T.G. Brock, \& D. Kim, Hormones and the orientation of growth, in P.J. Davies (Ed.), Plant Hormones, Second Edition, Dordrecht, The Netherlands: Kluwer Academic Publishers, 547-571.

[13] J.M. Kimbrough, R. Salinas-Mondragon, W.F. Boss, C.S. Brown, \& H.W. Sederoff, The fast and transient transcriptional network of gravity and mechanical stimulation in the Arabidopsis root apex, Plant Physiology, 136(1), 2004, 27902805.

[14] J. Lichtenberg, E. Jacox, J.D. Welch, K. Kurz, X. Liang, M.Q. Yang, F. Drews, K. Ecker, S.S. Lee, L. Elnitski, \& L.R. Welch, Word-based characterization of promoters involved in human DNA repair pathways, BMC Genomics, 10(Suppl 1), 2009, S18.

[15] J. Lichtenberg, A. Yilmaz, J.D. Welch, K. Kurz, X. Liang, F. Drews, K. Ecker, S.S. Lee, M. Geisler, E. Grotewold, \& R.W. Lonnie, The word landscape of the non-coding segments of the Arabidopsis thaliana genome, BMC Genomics, 10, 2009 , 463.

[16] S.K. Palaniswamy, S. James, H. Sun, R.S. Lamb, R.V. Davuluri, \& E. Grotewold, AGRIS and AtRegNet: A platform to link cisregulatory elements and transcription factors into regulatory networks, Plant Physiology, 140(3), 2006, 818-829.

[17] R.V. Davuluri, H. Sun, S.K. Palaniswamy, N. Matthews, C. Molina, M. Kurtz, \& E. Grotewold, AGRIS: Arabidopsis Gene Regulatory Information Server, an information resource of Arabidopsis cis-regulatory elements and transcription factors, BMC Bioinformatics, 4(1), 2003, 25.

[18] E. Wingender, X. Chen, R. Hehl, H. Karas, \& I. Liebich, TRANSFAC: An integrated system for gene expression regulation, Nucleic Acids Research, 28, 2000, 316-319.

[19] J.C. Bryne, E. Valen, M.H. Tang, T. Marstrand, \& O. Winther, JASPAR, the open access database of transcription factorbinding profiles: New content and tools in the 2008 update, Nucleic Acids Research, 36, 2008, D102-106.

[20] H.M. Hsu, S.J. Ong, M.C. Lee, \& J.H. Tai, Transcriptional regulation of an iron-inducible gene by differential and alternate promoter entries of multiple myb proteins in the protozoan parasite Trichomonas vaginalis, Eukaryotic Cell, 8(3), 2009, 362-372.

[21] J.H. Yoo, C.Y. Park, J.C. Kim, W.D. Heo, M.S. Cheong, H.C. Park, M.C. Kim, B.C. Moon, M.S. Choi, Y.H. Kang, J.H. Lee, H.S. Kim, S.M. Lee, H.W. Yoon, C.O. Lim, D.J. Yun, S.Y. Lee, W.S. Chung, \& M.J. Cho, Direct interaction of a divergent CaM isoform and the transcription factor, MYB2, enhances salt tolerance in arabidopsis. The Journal of Biological Chemistry, 280(5), 2005, 3697-3707.

[22] R.C. Gentleman, V.J. Carey, D.M. Bates, B. Bolstad, M. Dettling, S. Dudoit, B. Ellis, L. Gautier, Y.C. Ge, J. Gentry, K. Hornik, T. Hothorn, W. Huber, S. Lacus, R. Irizarry, F. Leisch, C. Li, M. Maechler, A.J. Rossini, G. Sawitzki, C. Smith, G. Smyth, L. Tierney, J.Y.H. Yang, \& J.H. Zhang, Bioconductor: open software development for computational biology and bioinformatics, Genome Biology, 5(10), 2004, R80.

[23] Z. Wu, R.A. Irizarry, R. Gentleman, F. Martinez-Murillo, \& F. Spencer, A model-based background adjustment for oligonucleotide expression arrays, Journal of the American Statistical Association, 99(468), 2004, 909-917.

[24] R. Breitling, P. Armengaud, A. Amtmann, \& P. Herzyk, Rank products: A simple, yet powerful, new method to detect differentially regulated genes in replicated microarray experiments, FEBS Letter, 573(1-3), 2004, 83-92.

[25] L. Kaufman \& P.J. Rousseeuw, Finding groups in data (John Wiley \& Sons, Inc. 1990), 190, 191, 212, 217.
[26] T. Beissbarth \& T.P. Speed, GOstat: Find statistically overrepresented gene ontologies within a group of genes, Bioinformatics, 20(9), 2004, 1464-1465.

[27] B. Lenhard \& W.W. Wasserman, TFBS: Computational framework for transcription factor binding site analysis, Bioinformatics, 18, 2002, 1135-1136.

[28] E. Jacox \& L. Elnitski, Finding occurrences of relevant functional elements in genomic signatures, International Journal of Computational Science, 2(5), 2008, 599-606.

[29] C. Martin \& J. Paz-Ares, MYB transcription factors in plants, Trends in Genet, 13, 1997, 67-73.

[30] G.R. Teakle, I.W. Manfield, J.F. Graham, \& P.M. Gilmartin, Arabidopsis Thaliana GATA factors: Organisation, expression and DNA-binding characteristics, Plant Molecular Biology, $50(1), 2002,43-57$.

[31] U. Hartmann, W.J. Valentine, J.M. Christie, J. Hays, G.I Jenkins, \& B. Weisshaar, Identification of UV/blue lightresponse elements in the Arabidopsis thaliana chalcone synthase promoter using a homologous protoplast transient expression system, Plant Molecular Biology, 36, 1998 741-754.

[32] R. Solano, A. Stepanova, Q. Chao, \& J.R. Ecker, Nuclear events in Ethylene signaling: A transcriptional cascade mediated by ethylene-insensitive3 and ethylene-response-factor1, Genes of Development, 12, 1998, 3703-3714.

[33] J.J. Tilly, D.W. Allen, \& T. Jack, The CArG boxes in the promoter of the Arabidopsis floral organ identity gene APETALA3 mediate diverse regulatory effects, Development, 125(9), 1998, 1647-1657

[34] C. Despres, C. Delong, S. Glaze, E. Liu, \& P.R. Fovert, The Arabidopsis NPR1/NIM1 protein enhances the DNA binding activity of a subgroup of the TGA family of bZIP transcription factors, Plant Cell, 12, 2000, 279-290.

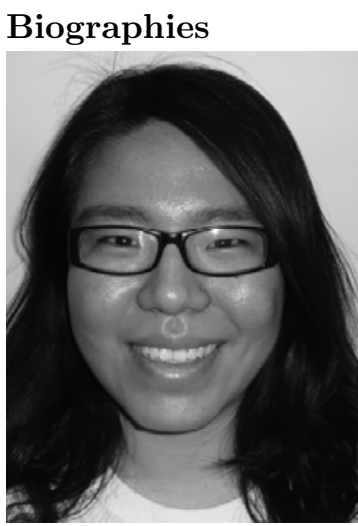

Xiaoyu Liang received her B.S. degree in management information system from University of Shanghai for Science and Technology, Shanghai, China in 2006. She is currently a master student in Bioinformatics at Ohio University, USA. Her research interest is cis-regulatory module discovery.

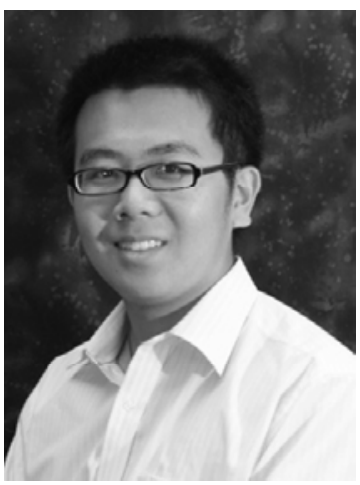

Kaiyu Shen received his B.S. degree in molecular and cellular biology from the University of Science and Technology of China, Hefei, China in 2006. He is currently a Ph.D. candidate in Molecular and Cellular Biology at Ohio University. His research interests are gravitropism and microarray data analysis. 


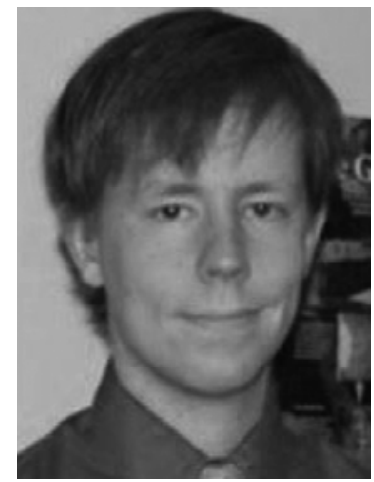

Jens Lichtenberg received his B.Sc. and M.Sc. degrees in Business Informatics from the Clausthal University of Technology, Germany in 2002 and 2004, respectively. He is currently a Ph.D. candidate in Bioinformatics at Ohio University, USA, and the President of the Regional Student Group Ohio for the Student Council of the International Society of Computational Biology. His research interests include regulatory genomics and proteomics.

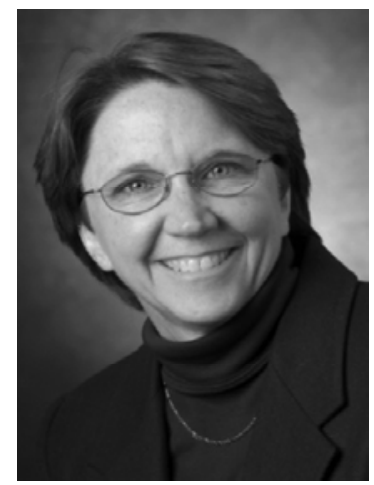

Sarah Wyatt is an associate professor of Plant Developmental Biology at Ohio University. She received her Ph.D. degree in Plant Physiology and Molecular Biology at Purdue University. Her main research interests are plant gravitropism, signal transduction, and bioinformatic analysis of genomic data. She is also faculty advisor for the DNA Analysis/ genomics facility at Ohio University.

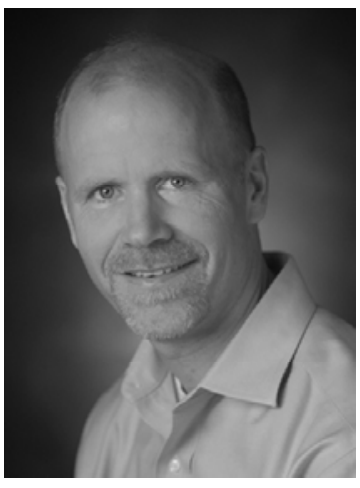

Lonnie Welch received his Ph.D. degree in Computer and Information Science from the Ohio State University. $\mathrm{He}$ is the Stuckey Professor of Electrical Engineering and Computer Science at Ohio University, and is a member of the Graduate Faculties of the Biomedical Engineering Program and of the Molecular and Cellular Biology Program. He directs the Bioinformatics Laboratory, where he performs research in the area of computational genomics. His research has been sponsored by the Defense Advanced Research Projects Agency, the Navy, NASA, the National Science Foundation, the Army, and the Ohio Board of Regents. 